\title{
CMB anisotropy and BBN constraints on pre-recombination decay of dark matter to visible particles
}

\author{
Sandeep Kumar Acharya, ${ }^{a}$ Rishi Khatri ${ }^{a}$ \\ ${ }^{a}$ Department of Theoretical Physics, Tata Institute of Fundamental Research, Mumbai 400005, India \\ E-mail: sandeepkumar@theory.tifr.res.in, khatri@theory.tifr.res.in
}

\begin{abstract}
Injection of high energy electromagnetic particles around the recombination epoch can modify the standard recombination history and therefore the CMB anisotropy power spectrum. Previous studies have put strong constraints on the amount of electromagnetic energy injection around the recombination era (redshifts $z \lesssim 4500$ ). However, energy injected in the form of energetic $(>\mathrm{keV})$ visible standard model particles is not deposited instantaneously. The considerable delay between the time of energy injection and the time when all energy is deposited to background baryonic gas and CMB photons, together with the extraordinary precision with which the CMB anisotropies have been measured, means that $\mathrm{CMB}$ anisotropies are sensitive to energy that was injected much before the epoch of recombination. We show that the CMB anisotropy power spectrum is sensitive to energy injection even at $z=10000$, giving stronger constraints compared to big bang nucleosynthesis and CMB spectral distortions. We derive, using Planck CMB data, the constraints on long-lived unstable particles decaying at redshifts $z \lesssim 10000$ (lifetime $\tau_{X} \gtrsim 10^{11}$ s) by explicitly evolving the electromagnetic cascades in the expanding Universe, thus extending previous constraints to lower particle lifetimes. We also revisit the BBN constraints and show that the delayed injection of energy is important for $\mathrm{BBN}$ constraints. We find that the constraints can be weaker by a factor of few to almost an order of magnitude, depending on the energy, when we relax the quasi-static or on-the-spot assumptions.
\end{abstract}




\section{Introduction}

The incredible precision measurement of the cosmic microwave background (CMB) anisotropy power spectrum allows us to not just measure the 6 parameters of the standard $\Lambda \mathrm{CDM}$ cosmological model [1] with high precision but also study extensions to it and constrain new physics beyond the standard model of particle physics. The CMB is sensitive to new physics such as dark matter self interactions [2, 3], dark matter-dark radiation interactions [4, 5], decay of long-lived unstable particles [6,7], evaporating primordial black holes [8, 9], and annihilation of dark matter to standard model particles [10-12]. The physics in the dark sector can affect CMB both gravitationally $[5,13,14]$ and electromagnetically $[6,7,9,10]$. In particular, there are many particle physics motivated scenarios where dark matter can annihilate or decay to standard model particles [6, 15-22], see [23, 24] for reviews.

In this paper, we are interested in new physics that can inject electrons, positrons and photons with energy much greater than the CMB temperature into the primordial plasma. We will focus on the decay of dark matter ( or new long-lived unstable particles) but our general conclusions about the ability of CMB to constrain energy injection at high redshifts are applicable to other processes like evaporating black holes as well. Energetic electrons, positrons, and photons, injected by new physics, deposit their energy in the background baryon-photon plasma by heating background electrons and by ionization and excitation of neutral atoms. A fraction of energy escapes as low energy photons below $10.2 \mathrm{eV}$ (rest frame Lyman-alpha threshold) [10, 25] resulting in spectral distortion of the CMB. Increased ionization rate of neutral hydrogen and helium due to energy injection around recombination results in higher freeze-out number density of residual free electrons after recombination compared to the standard recombination [26, 27]. Increased number of scatterings of CMB photons with free electrons damp the temperature anisotropy while giving a boost to polarization signal $[6,7,10,28,29]$. Precision measurement of the CMB anisotropy spectrum therefore puts constraints on the amount of electromagnetic energy that can be injected during the epoch of recombination and hence on the parameters of the new physics such as the annihilation cross-section of dark matter, fraction of decaying dark matter and lifetime of decaying particles [12, 30, 31], and abundance of primordial evaporating black holes as a function of black hole mass [9].

Energy deposition for sub-keV electrons to a hydrogen and helium gas as a function of ionization fraction has been studied in $[32,33]$. Monte Carlo simulation of higher energy electrons including inverse Compton scattering (ICS) was done in [34]. In these calculations, the high energy $(\gtrsim \mathrm{keV}$ ) electrons are evolved until their energy drops down to $\sim \mathrm{eV}$, after depositing most of their energy to background particles, at which point the rest of their energy is deposited as heat. Keeping track of the evolution history from injected energy to $\sim \mathrm{eV}$ energy for each injected particle makes these calculations computationally expensive. An alternative recursive solution to the above problem was provided in $[10,35,36]$, who

also included relativistic processes of electron-positron pair production and photon-photon elastic scattering. In this method, the pre-computed energy deposition history of lower energy 
particles is used to compute energy deposition of higher energy particles. Using this recursive method, the authors in [30] have calculated the constraints on fraction of decaying dark matter (with respect to total dark matter) for decay to monochromatic electron-positron and photon pairs as a function of dark matter mass $\left(m_{X}\right)$ and lifetime $\left(\tau_{X}\right)$ for $\tau_{X} \gtrsim 10^{13} \mathrm{~s}$ (or corresponding decay redshift $z_{X} \lesssim 1200$ ) using Planck 2015 [37] CMB anisotropy power spectrum data. Similar constraints have been provided in [31], where the authors have used the publicly available results of energy deposition fractions from [38] to constraint particle lifetimes $\tau_{X} \gtrsim 10^{12} \mathrm{~s}\left(z_{X} \lesssim 4500\right)$. The authors in [31] also provide an effective on-the-spot ansatz, which means the energy injected at a particular redshift is immediately deposited at that redshift. They absorb the beyond on-the-spot corrections into an effective energy injection history function. Their results show that the constraints on decaying dark matter abundance with this on-the-spot ansatz agrees well with the full calculations of [30] for $\tau_{X} \gtrsim$ $10^{13} \mathrm{~s}$ but not for lower lifetimes.

The high energy particles that are injected into the plasma do not deposit all of their energy into heating, excitation, ionization and sub- $10.2 \mathrm{eV}$ photons instantaneously but over a period of time. This delay between the energy injection and deposition implies that the $\mathrm{CMB}$ anisotropies can be affected by energy that was injected much before the epoch of recombination. We have developed a new code for evolution of high energy particle cascades in the expanding Universe based on the method proposed in [35, 36]. We calculate the constraints on dark matter decaying to monochromatic electron-positron and photon pairs by evolving the full electromagnetic cascade, starting from the initial $\mathrm{keV}-\mathrm{TeV}$ high energy electrons, positrons and photons until $\mathrm{eV}$ energies, i.e. until all of the energy has been deposited or escapes as CMB spectral distortions. Our code takes into account all relevant processes at all energies and thus presents a unified approach without separate treatments for the low and high energy parts of the cascade. We provide CMB anisotropy constraints, using Planck 2015 [37] data, for lower lifetimes than what has been studied before, upto the point where the constraints from big bang nucleosynthesis (BBN) and spectral distortions become stronger. We will see below that the $\mathrm{CMB}$ anisotropies give the strongest constraints for energy injection at redshifts as high as $z \approx 10000$. We will show this in the specific context of decay of long-lived unstable particles, but this conclusion will be true for any general energy injection scenario.

The high energy photons in the cascade can also destroy primordial elements produced in the BBN, changing their abundances [39-45]. We revisit the BBN constraints in section 6. We will see that the delay between the energy injection and deposition is important for the BBN constraints also and results in weakening of constraints compared to the current constraints in literature which use instantaneous deposition approximation.

\section{Physics of electromagnetic cascade in an expanding Universe}

Any injected electromagnetic energy is deposited in the fully ionized baryonic gas as heat while for the partially neutral gas, a fraction of energy goes into excitation and ionization 
of atoms. In addition, a significant fraction of energy can escape as low energy photons with energy less than $10.2 \mathrm{eV}$ (Lyman-alpha threshold in the local rest frame) [25]. These photons will show up as spectral distortion in the CMB spectrum but take no part in the CMB anisotropy power spectrum analysis. We include the energy loss to these photons in the total deposited energy fraction to keep track of energy conservation.

Energy is deposited in the baryonic gas through various atomic collision processes. A photon with energy, $E_{\gamma}>13.6 \mathrm{eV}$, can photo-ionize a neutral hydrogen atom creating a free electron with excess energy $E_{\gamma}-13.6 \mathrm{eV}$ going into the kinetic energy of the free electron. This electron, if it has kinetic energy greater than $13.6 \mathrm{eV}$, can scatter with neutral atoms, ionizing them, and producing secondary free electrons or excite electrons in neutral atoms from ground to higher energy levels. It can also scatter with free electrons to deposit its kinetic energy as heat through Coulomb scattering. These atomic processes are efficient for an injected electron with energy $\lesssim \mathrm{keV}$. For a higher energy electron, ICS process becomes the dominant process. By this process the electron boosts CMB photons, producing a spectrum of high energy photons at the expense of its kinetic energy. These photons can then ionize neutral atoms or lose their energy by scattering with background electrons through Compton scattering. Compton scattering of high energy photons with bound electrons can transfer sufficient energy through electron recoil to ionize them. At even higher energy, photons can pair produce electron-positron pairs on scattering with background electrons, ions, neutral atoms and CMB photons or scatter elastically with CMB photons. These high energy particles create numerous energetic particles by boosting background particles. Thus, as the cascade progresses, the energy of injected particle is shared by more and more particles with energy of each particle in the cascade decreasing until all of the energy is deposited as heat, excitation, ionization, or escapes as low energy photons. Thus, a high energy particle (>> $\mathrm{keV}$ ) deposits its energy by producing a lot of sub-keV secondary particles while direct deposition is negligible. Since energetic electrons, positrons and photons are produced cyclically ( photons boosting background electrons or pair-producing electrons-positrons and the high energy electrons and positrons boosting CMB photons), one has to evolve the electron, positron and photon spectra simultaneously. Electrons and positrons produced at one timestep can deposit their energy to baryonic gas or CMB photons at that timestep as their collisional rates are much higher compared to Hubble expansion rate $(H(z))$. But for photons, the collisional rates at high energy are comparable to Hubble rate for $z \lesssim 10000[10,46]$. Therefore, we must evolve the photon spectrum with background expansion taken into account. More importantly, high energy photons deposit their energies on timescales much larger than the Hubble time $\left(t_{H}=1 / H(z)\right)$ and thus act as messengers of energy injection carrying information from redshifts as high as $z \approx 10000$ to the recombination epoch, where they influence the CMB anisotropies.

A positron's energy loss mechanism is identical to that of an electron with the exception that it can annihilate with a background electron. Therefore, each injected positron can be approximated by an electron with the same kinetic energy plus two $511 \mathrm{keV}$ photons $[38,46]$. 
From the above discussion, it is clear that the calculations can be approximately

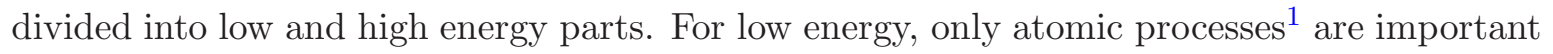
while for high energy, atomic processes are negligible and only high energy relativistic processes are important. The energy deposition of sub-keV particles to background particles is a function of the ionization fraction of background baryonic gas. This strategy, to separate out the low and high energy physics parts, was used in calculations of $[10,38]$. They use tabulated results of energy deposition fractions for sub-keV particles as a function of ionization fraction of baryonic gas from monte-carlo calculations [34], which forms the low energy code. The high energy code evolves the particles with all high energy scattering processes giving the spectra of low energy particles at each timestep. These low energy particles are removed from high energy code and fed to low energy code to calculate the energy deposition fractions at that timestep. The interface between low energy to high energy code was chosen to be $3 \mathrm{keV}$. The results are however sensitive to this transition energy. Changing this interface slightly to $1 \mathrm{keV}$ makes a difference of few percent while changing it to $10 \mathrm{keV}$ or few eV can result in much bigger difference see (Fig. 1 and 2 of [38]).

In this work, we present a unified calculation to solve for energy deposition fraction and evolution of electromagnetic cascade simultaneously, taking into account all relevant physics without an arbitrary division between low and high energy physics. In particular, we do not rely on monte-carlo calculations in a static Universe of sub-keV energy deposition, but evolve the sub-keV particles also in the expanding Universe in the same code that evolves the high energy cascade. We follow the formalism of $[35,36]$ and divide the energy range from $\mathrm{eV}$ to $\mathrm{TeV}$ in 200 logarithmically spaced bins. The problem of electromagnetic cascade in an expanding Universe then reduces to that of a system of coupled ordinary differential equations for different particles and energy bins. Energetic particles always lose energy to background particles and move from high energy bins to lower energy bins. We, therefore, start by first calculating the cascade of lowest energy bin and successively move to higher energy bins. For injection of a particular particle, the subsequent cascade of lower energy particles created by it, can be reused from previous steps. The lower energy cascades computed once, are thus used over and over again, thereby making these calculations fast. Interactions in-between high energy injected particles can be neglected as their number density is much smaller compared to the number density of background particles. Formally, we have reduced the problem of evolution of electromagnetic cascade to solving a set of coupled linear algebraic equations which are triangular and we are solving these equations by back-substitution $[35,36,46]$. The cross-sections for various high energy processes used in this paper are given in the appendix of [46]. We give the cross-sections for atomic processes included in this paper in Appendix A. We refer the reader to [46] for further technical details of the algorithm. We briefly review a few important aspects of energy deposition in the next two subsections.

\footnotetext{
${ }^{1}$ We club low energy Coulomb scattering also into atomic processes for this discussion
} 


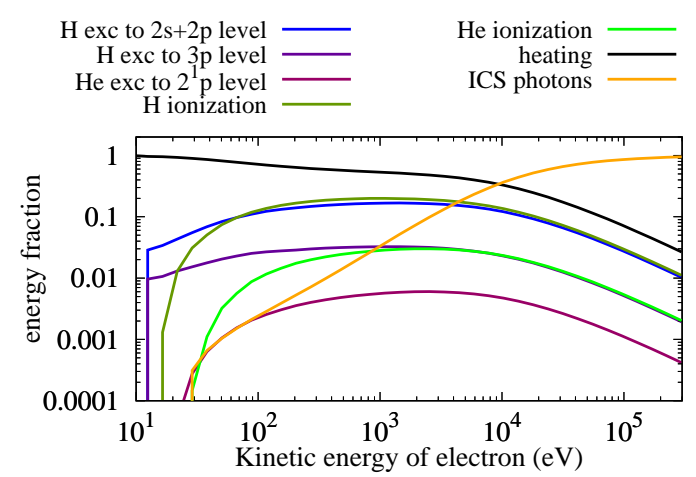

(a) $x_{\mathrm{H}}=0.04, x_{\mathrm{He}} \sim 0, \mathrm{z}=1000$.

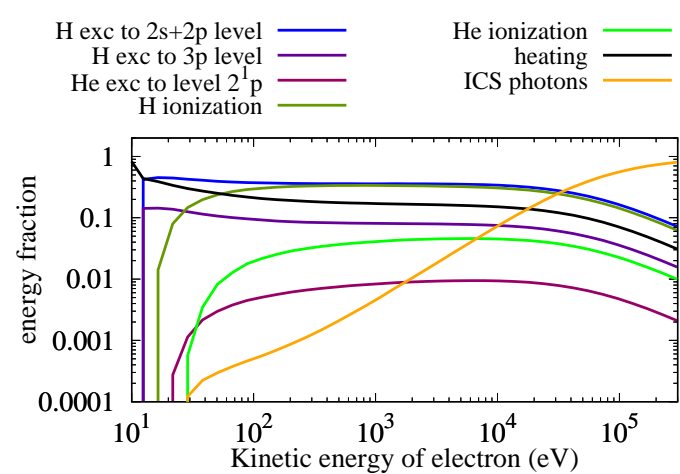

(b) $x_{\mathrm{H}}=0.0002, x_{\mathrm{He}} \sim 0, \mathrm{z}=100$

Figure 1: Electron injection: Fraction of energy deposited through heating, excitation, ionization or CMB photons (Including sub-10.2 eV and higher energy photons) as a function of electron kinetic energy with the hydrogen $\left(x_{\mathrm{H}}\right)$ and helium $\left(x_{\mathrm{He}}\right)$ ionization fractions from Recfast ++ [47]. Electron energy is deposited instantaneously as their energy loss rate is much faster than the Hubble rate (on-the-spot approximation). ICS photons include both sub-10.2 eV and higher energy photons. The photons with energy greater than $10.2 \mathrm{eV}$ are processed in next timestep of the cascade evolution.

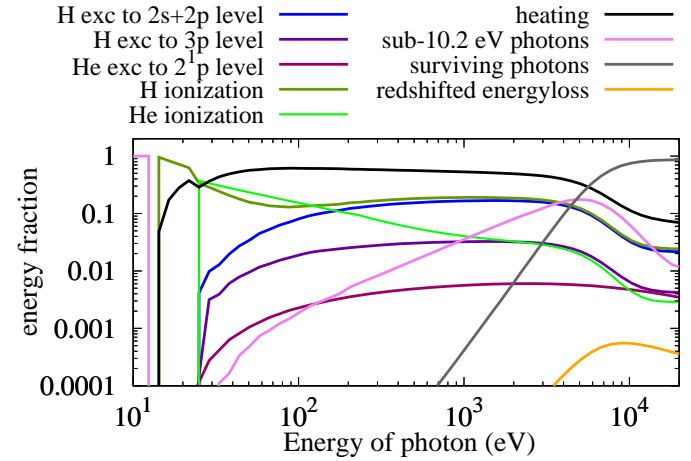

(a) $x_{\mathrm{H}}=0.04, x_{\mathrm{He}} \sim 0, \mathrm{z}=1000$.

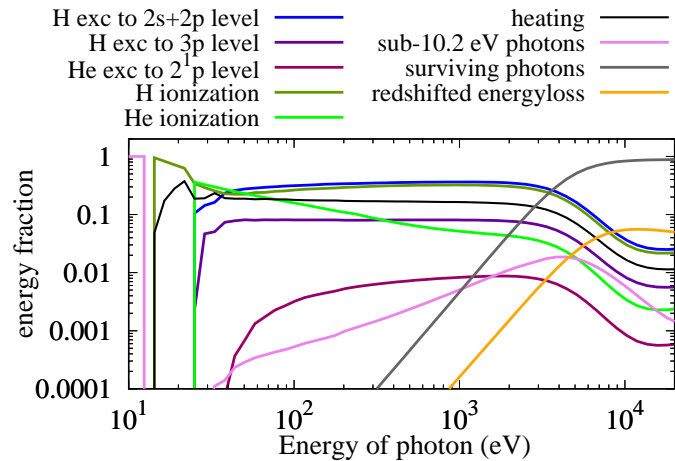

(b) $x_{\mathrm{H}}=0.0002, x_{\mathrm{He}} \sim 0, \mathrm{z}=100$

Figure 2: Photon injection: Fraction of energy deposited through heating, excitation, ionization and sub-10.2 eV photons, redshifted energy loss and surviving photons as a function of photon energy.

\subsection{Electron injection}

In Fig. 1, we plot fractions of energy deposited instantly (on the timescale much smaller than the Hubble time) through heating, excitation, ionization and to CMB photons by ICS as a function of the kinetic energy of the injected electron at $\mathrm{z}=1000$ and 100 (with the ionization fractions calculated from Recfast $++[47,48])$. Helium ionization fraction is extremely small at these redshifts. We see the deposited fractions as a function of energy are somewhat flat 
in both plots at $\sim \mathrm{keV}$ energies, explaining the relative insensitivity of the choice of interface energy around $\sim \mathrm{keV}$ between low and high energy code of [38]. ICS is not important at low energies but becomes dominant at $\gtrsim 10 \mathrm{keV}$. The crossover between ICS and the atomic processes is redshift dependent. We can see the crossover at $\mathrm{z}=100\left(\sim 10^{4} \mathrm{keV}\right)$ to be at higher electron kinetic energy than at $\mathrm{z}=1000\left(\sim 10^{3} \mathrm{keV}\right)$. An electron with Lorentz factor $\gamma$ can boost a photon with energy $\epsilon$ to $\sim \gamma^{2} \epsilon$ through ICS. Therefore, the energy loss rate of an electron by ICS $(\propto \epsilon \propto 1+z)$ is higher at higher redshifts. For a partially ionized Universe, the dominant channel of energy deposition is through heating. Fractions of energy deposited by electrons through excitation to second level and ionization are similar because the scattering cross-sections for these processes are of similar magnitude. Excitation to higher energy levels becomes approximately one order of magnitude smaller for each increase in level as the cross-sections become smaller. Energy deposited to helium is smaller by a factor of $\sim 10$ compared to hydrogen just because the ratio of number density of helium to hydrogen $f_{\mathrm{He}} \approx 0.08$, making helium an unlikely target for an incident electron. These qualitative statements are true even when the injected particles have energies of the order $\mathrm{GeV}-\mathrm{TeV}$, since these particles deposit their energy by first producing lots of $\sim \mathrm{keV}$ secondary particles.

\subsection{Photon injection}

In Fig. 2, we plot the fraction of energy deposited by a photon to baryonic gas by atomic processes, energy-loss to sub-10.2 eV photons, redshifted energy loss, and surviving photons for energy injection at $z=100$ and 1000 as a function of photon energy. These energy fractions are calculated by first comparing the cooling rate of the injected photon, including all scattering processes, with the Hubble rate at a particular redshift, $H(z)$. The cooling time of photons is given by, $t_{\text {cool }}=1 /\left(d \ln E_{\gamma} / d t\right)[10,46]$, where $\mathrm{d} \ln E_{\gamma} / \mathrm{d} t$ is the total energy loss rate of photons due to scattering with the background particles and $t$ is the proper time. Therefore, the fraction of energy lost and deposited by a photon, ionizing neutral gas and boosting free electrons, is given by the ratio $\frac{t_{\text {cool }}^{-1}}{t_{\text {cool }}^{-1}+H(z)}$, while rest of the energy survives. The free electrons created during these processes deposit their energy immediately according to the discussion in the previous section. Since a large fraction of the photon energy is deposited by first boosting electrons, it explains the similarity of the energy deposition fractions by electrons and photons for $\sim \mathrm{keV}$ energies. Close to the threshold of ionization of neutral hydrogen (13.6 $\mathrm{eV}$ ) and neutral helium $(24.6 \mathrm{eV})$, the dominant fraction of energy goes into ionization. At higher energy, a photon has a higher chance to survive since the high energy collision rates for photons and the Hubble rate are of the same order. These surviving photons then redshift to the next timestep. 


\section{Modification to the recombination history from electromagnetic energy injection}

The problem of cosmological recombination has been studied in great detail beginning with the first calculations of $[26,27]$. The need for multilevel calculations was emphasized by [49] for precision calculations needed by the CMB experiments in the 21st century. There has been tremendous progress since then, culminating in the fast effective multilevel recombination codes $[47,50-56]$. Most of the complicated dynamics of recombination can be captured by the effective 3-level atoms originally proposed by [26, 27], with the equations suitably modified by a fudge factor [48] or a fudge function [47]. In these calculations, only the first excited levels with the principal quantum number $n=2$ of hydrogen and helium are resolved, in addition to the ground state and the continuum or the ionized state of the atom. However, the energetic electrons in the electromagnetic cascade can also excite the hydrogen and helium atoms to $n=2$ as well as higher energy levels. To take these excitations into account requires us to resolve the higher levels. The energy injection modifies the standard recombination calculations by adding extra source or sink terms to the differential equations governing the population of different levels [57]. In particular, the equations for the ground states of hydrogen and helium get an extra sink term due to direct ionizations from the ground state,

$$
\begin{aligned}
& \left.\frac{\mathrm{d} x_{\mathrm{H}_{1 \mathrm{~s}}}}{\mathrm{~d} t}\right|_{\mathrm{inj}}=-f_{\mathrm{Hi}}(z) \frac{\mathrm{d} E_{\mathrm{inj}} / \mathrm{d} t}{n_{\mathrm{H}} E_{\mathrm{H}_{1 \mathrm{~s}}}} \\
& \left.\frac{\mathrm{d} x_{\mathrm{He}}}{\mathrm{d} t}\right|_{\mathrm{inj}}=-f_{\mathrm{Hei}}(z) \frac{\mathrm{d} E_{\mathrm{inj}} / \mathrm{d} t}{n_{\mathrm{H}} E_{\mathrm{He}_{1 \mathrm{~s}}}},
\end{aligned}
$$

where $x_{\mathrm{H}_{1 \mathrm{~s}}}=n_{\mathrm{H}_{1 \mathrm{~s}}} / n_{\mathrm{H}}, x_{\mathrm{He}_{1 \mathrm{~s}}}=n_{\mathrm{He}_{1 \mathrm{~s}}} / n_{\mathrm{H}}, n_{\mathrm{H}}$ is the total (ionized + neutral) hydrogen number density, $n_{\mathrm{H}_{1 \mathrm{~s}}}$ and $n_{\mathrm{He}_{1 \mathrm{~s}}}$ are the number densities of neutral hydrogen and helium atoms respectively, $E_{\mathrm{H}_{1 \mathrm{~s}}}$ and $E_{\mathrm{He}_{1 \mathrm{~s}}}$ are the binding energies for hydrogen and helium respectively, $d E_{\mathrm{inj}} / d t$ is the rate of injection of energy density, and $f_{\mathrm{Hi}}$ and $f_{\mathrm{Hei}}$ are the fractions of injected energy going into hydrogen and helium ionizations respectively. Similar terms are also added for excitations to the equations for the levels involved in the respective transitions [57]. Note that we do not assume that the ionization energy is divided among the hydrogen and helium according to their relative number density but calculate the respective fractions explicitly during the cascade evolution. The energy deposition fractions $f_{i}(z)$, where $i$ labels the different channels such as hydrogen and helium ionization, excitations etc, depends upon the redshift of injection, the energy of the injected particle, and whether the injected particle is an electron, positron or photon.

We also take into account the heating of background baryonic gas due to energy injection by adding a source term to baryon temperature $\left(T_{\mathrm{b}}\right)$ evolution equation [25-27],

$$
(1+z) \frac{\mathrm{d} T_{\mathrm{b}}}{\mathrm{d} z}=\frac{8 \sigma_{\mathrm{T}} a_{\mathrm{R}} T_{\mathrm{CMB}}^{4}}{3 m_{\mathrm{e}} c H} \frac{x_{\mathrm{e}}}{1+f_{\mathrm{He}}+x_{\mathrm{e}}}\left(T_{\mathrm{b}}-T_{\mathrm{CMB}}\right)-\frac{2}{3 k_{\mathrm{B}} H} \frac{K_{h}}{1+f_{\mathrm{He}}+x_{\mathrm{e}}}+2 T_{\mathrm{b}},
$$

where $x_{\mathrm{e}}=n_{\mathrm{e}} / n_{\mathrm{H}}, n_{\mathrm{e}}$ is the free electron number density, $\sigma_{\mathrm{T}}$ is the Thomson cross section, $T_{\mathrm{CMB}}$ is the $\mathrm{CMB}$ temperature, $a_{\mathrm{R}}$ is the radiation constant, $m_{\mathrm{e}}$ is the mass of electron, $k_{\mathrm{B}}$ 
is the Boltzmann constant, $c$ is the speed of light, $K_{h}=f_{h} \frac{\left(\mathrm{d} E_{\mathrm{inj}} / \mathrm{d} t\right)}{n_{\mathrm{H}}}$ parameterizes the heating from extra energy injection, and $f_{h}(z)$ is the fraction of injected energy used up in heating the baryonic gas. At $z \gtrsim 200$, the gas temperature and the CMB temperature are efficiently coupled due to Compton scattering between residual free electrons and CMB photons. As the CMB photons outnumber baryons by more than nine orders of magnitude, increasing the CMB temperature by even a small amount requires huge amount of energy injection. Since the fraction of energy going into ionization of neutral hydrogen and heating of baryonic gas for a high energy particle injection are of the same order, as shown in Figs. 1 and 2, this scenario would ionize the whole Universe, and is thereby ruled out by current data. We, therefore, expect the correction to baryonic temperature evolution to be of negligible importance compared to the ionization of neutral gas for CMB anisotropies. However, the corrections to baryonic temperature evolution can be important for $21 \mathrm{~cm}$ cosmology and reionization [58].

Electromagnetic energy injection can ionize singly ionized helium as well. The recombination of doubly ionized helium to singly ionized helium is given by Saha equilibrium solution to a very good approximation and this approximation is implemented in recombination codes to make the codes faster. Since the first recombination of helium happens very early $(z \approx 6000)$, when the hydrogen is fully ionized, the extremely large Thomson optical depth makes the visibility function $[26,27]$ vanishingly small. We can therefore ignore the extra ionizations of helium when calculating the CMB anisotropies. However, the extra ionizations and deviation from the Saha solution are important for recombination line spectral distortions [59]. We should clarify that we take both neutral and singly ionized helium into account when evolving the electromagnetic cascades and calculating the energy deposit fractions as explained in the previous section.

\subsection{Effects of excitations}

The extra ionizations from energy injection can have two contributions: (i) direct ionization from the ground level and (ii) excitation to higher levels and subsequent photo-ionization by CMB photons. As we saw in the previous section, the energy fraction going into excitation is comparable to the energy fraction going into direct ionization from the ground state. An excited atom will however not necessarily get ionized. It can also de-excite to a lower level, emitting a recombination line photon which can escape or, if it is a Lyman-series photon, be reabsorbed. We plot, in Fig. 3, the ratio of net de-excitation rate $P_{i}^{\text {esc }} A_{i}$, where $P_{i}^{\text {esc }}$ is the escape probability for the emitted photon $[26,27,47,48,56]$ and $A_{i}$ is the spontaneous transition rate, to the ionization rate for different levels $i$ as a function of redshift. For the first excited levels of hydrogen, photo-ionization dominates de-excitation at $z \gtrsim 1000$. At these redshifts, however, free electrons are likely to recombine with protons due to their high number density. There is, thus, almost no modification to the recombination history making the CMB anisotropy power spectrum insensitive to extra ionizations from the energy that is absorbed at these high redshifts. At lower redshifts, the CMB power spectrum is sensitive to the increase in the residual electron fraction due to extra ionizations. However at 


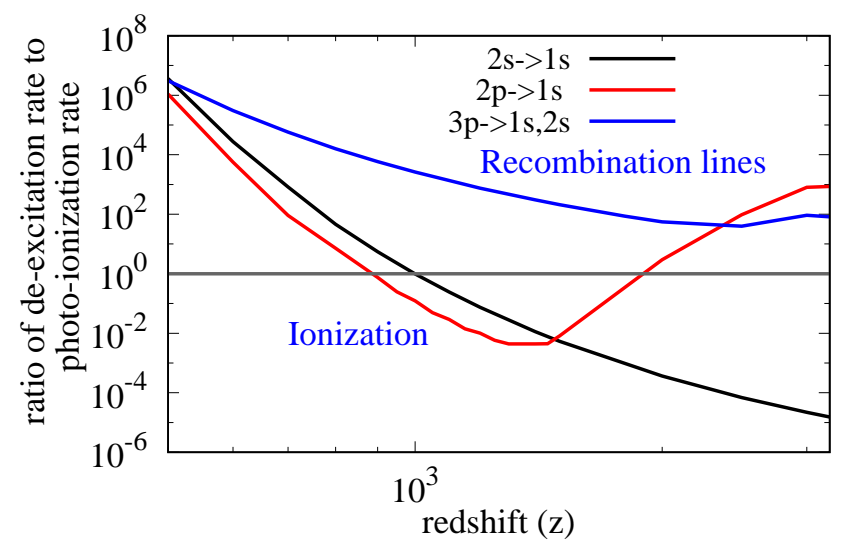

Figure 3: Ratio of net de-excitation rate $(2 \mathrm{~s}, 2 \mathrm{p} \rightarrow 1 \mathrm{~s}, 3 \mathrm{p} \rightarrow 1 \mathrm{~s}, 2 \mathrm{~s}$, after taking into account the escape probability) to photo-ionization rate from excited levels of hydrogen as a function of redshift. We have labeled regions where the contribution to ionization is important vs where most of the excitation energy goes into recombination lines.

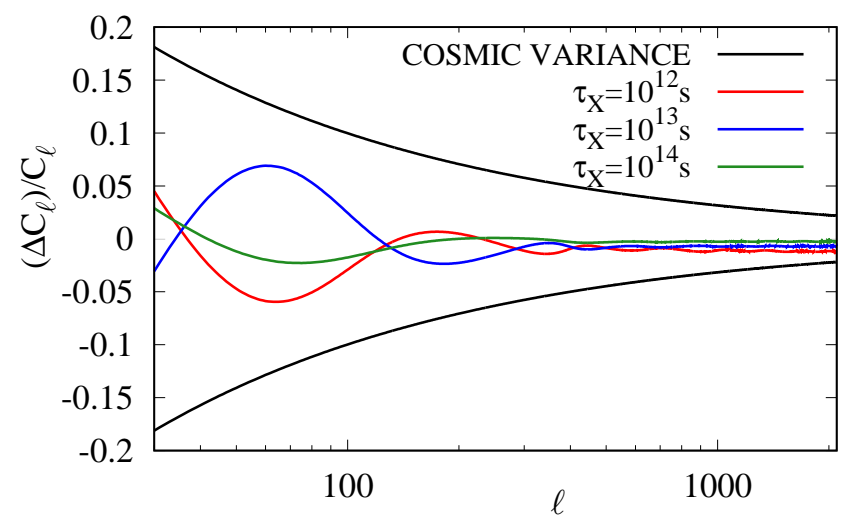

Figure 4: Fractional difference in the CMB angular power spectrum $\Delta C_{\ell} / C_{\ell}$, where $\Delta C_{\ell}$ is the difference in angular power spectrum $C_{\ell}$ calculated using Recfast ++ (only direct ionizations from the energy injection included) and CosmoRec (including also extra excitations) for 1 $\mathrm{TeV} e^{-} e^{+}$injection. We show three different lifetimes and choose $f_{X}$ to be the upper limit (derived in this work) for the corresponding lifetime. Also shown is the cosmic variance $\sqrt{2 /(2 \ell+1)}$.

$z \lesssim 1000$, photo-ionization from the first few levels becomes much less likely compared to deexcitation as the CMB temperature decreases, as seen in Fig. 3. We, therefore, do not expect excitations to be important in increasing the post-recombination ionization fraction. Hence, extra excitations from energy injection are not important for CMB anisotropies. Most of the 
energy going into excitations will therefore go into the cosmological recombination spectrum.

We check this explicitly using CosmoRec code [47] to take into account contribution to excitations upto third level for hydrogen and second level of helium by explicitly solving for the excited states without the assumption of effective three level atom. We have used CosmoRec setting with 10 hydrogen and helium interface shells [55], 500 hydrogen levels and all of radiative transfer effects flags turned on. We include contribution from injected energy to excitations from the ground state to $2 \mathrm{~s}, 2 \mathrm{p}$, and $3 \mathrm{p}$ levels of hydrogen and $2^{1} \mathrm{p}$ level of helium. The fractional difference in the CMB angular power spectrum $C_{\ell}$ as a function of multipole $\ell$ between CosmoRec (with excitations) and Recfast ++ (without excitations) is shown in Fig. 4 and compared with the cosmic variance. We show the comparison for pre-recombination decay $\left(\tau_{X}=10^{12} \mathrm{~s}\right)$, decay during recombination $\left(\tau_{X}=10^{13} \mathrm{~s}\right)$, and a post recombination case $\left(\tau_{X}=10^{14} \mathrm{~s}\right)$ using the corresponding upper limits for decay fraction $f_{X}$ derived below in this work. We find that the difference is much smaller than the cosmic variance.

Henceforth, we use Recfast ++ , since it is much faster, with contribution from the energy injections to only direct ionizations included while calculating the recombination history.

To illustrate the main effects on the CMB anisotropies, we plot in Fig. 5 the recombination histories and the E-mode polarization power spectrum for energy injection from dark matter decay with lifetime $\left(\tau_{X}=10^{14} \mathrm{~s}\right)$ longer than recombination era. The main effect is a boost in the residual electron fraction, which changes by order unity or more, after recombination. We expect this change to affect modes entering the horizon between the recombination and the reionization epoch. This is most clearly and dramatically seen in the E-mode polarization spectrum. The E-mode polarization spectrum gets a boost for the mulitpoles $(\ell)$ between the first recombination peak and the reionization bump, with fractional change as large as $50 \%$. However, the absolute signal for these multipoles is still very small, because the ionization fraction $x_{\mathrm{e}} \ll 1$. Therefore, the CMB constraints will be driven by not these obvious signals but by more subtle changes in the rest of the CMB temperature and polarization power spectrum, where the actual signal is much higher. In particular, the percent level suppression, due to extra optical depth given by the enhanced residual electrons, of the high $\ell$ modes is important. We give the plots for smaller lifetimes as well as the CMB temperature power spectrum in Appendix C.

\section{Energy injection and deposition from dark matter decay}

Energy injection from dark matter decay can be parameterized by,

$$
\frac{\mathrm{d} E_{\mathrm{inj}}}{\mathrm{d} t}=\frac{f_{X}}{\tau_{X}} \rho_{c} c^{2}(1+z)^{3} e^{-t / \tau_{X}},
$$

where $f_{X}$ is the fraction of decaying dark matter w.r.t. total dark matter, $\tau_{X}$ is the lifetime of dark matter with the corresponding decay redshift $z_{X}$ defined for dark matter with lifetime shorter than the age of the Universe. Energy injected in a redshift interval $\Delta z$ or timestep, 


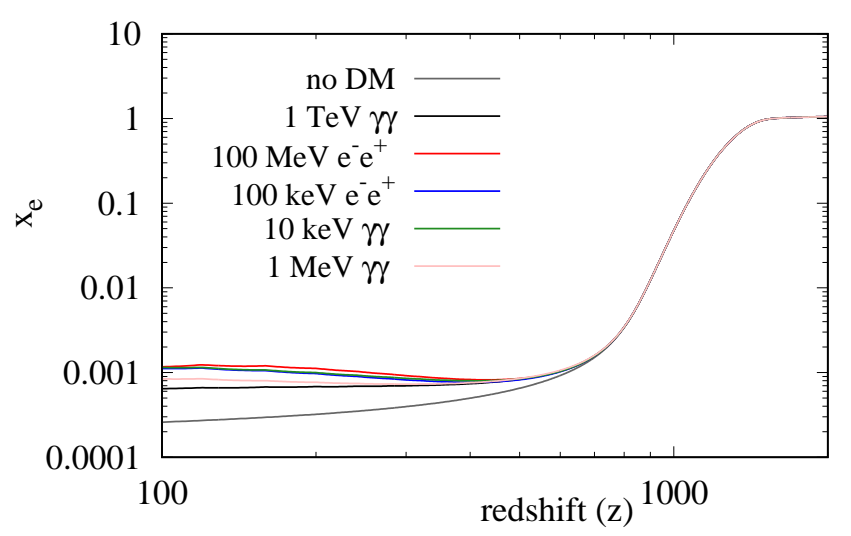

(a)

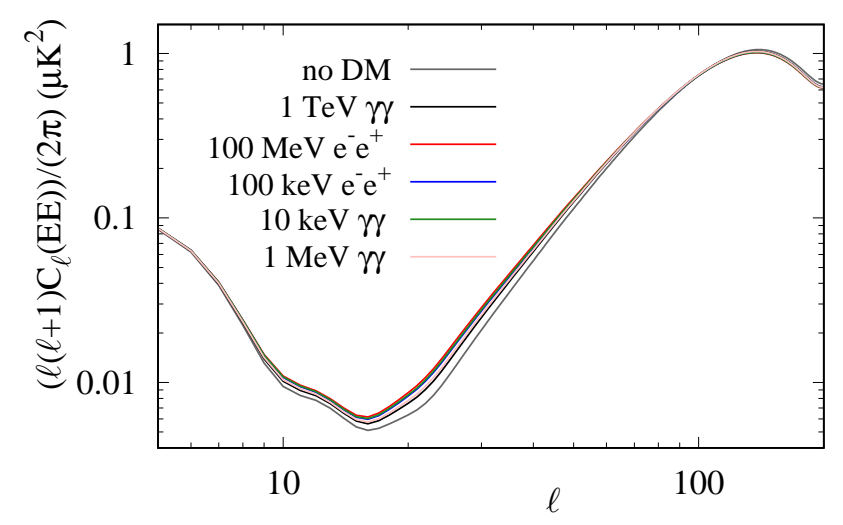

(c)

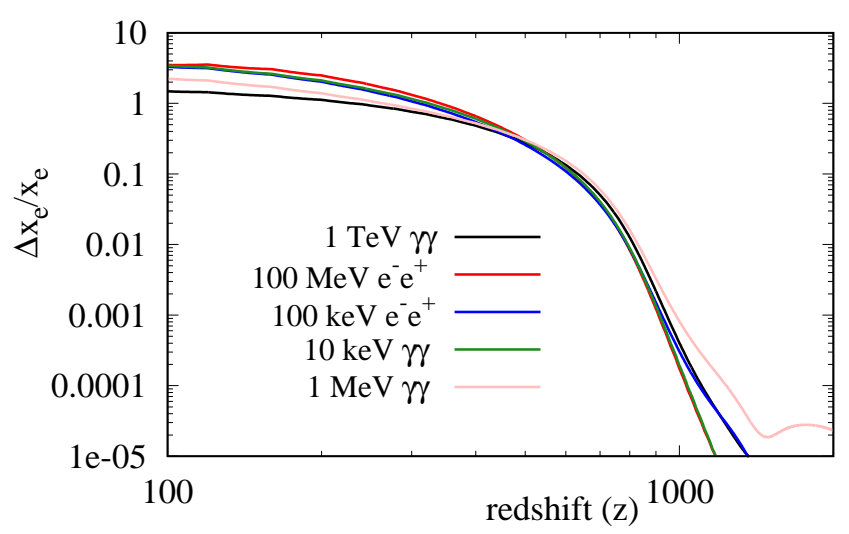

(b)

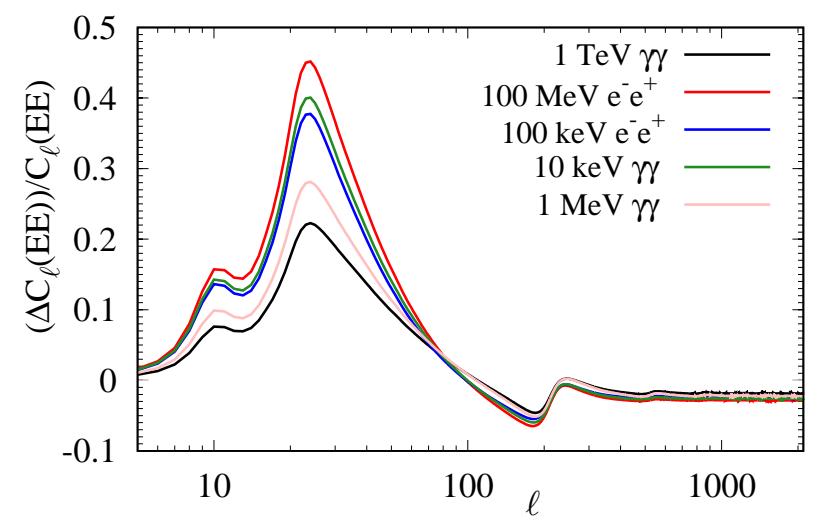

(d)

Figure 5: (a) Recombination history for different energy injection scenarios, (b) fractional change in ionization fraction, (c) CMB E-mode polarization spectrum, (d) fractional change in CMB E-mode power spectrum. All plots are for dark matter decay with lifetime $\left(\tau_{X}\right)=10^{14} \mathrm{~S}$ and fraction of decaying dark matter $\left(f_{X}\right)$ is equal to the $2-\sigma$ upper limits derived in this paper.

$\Delta t=\frac{|\Delta z|}{(1+z) H(z)}$, is given by,

$$
\Delta E_{\mathrm{inj}}=\frac{f_{X}}{\tau_{X}} \rho_{c} c^{2}(1+z)^{3} e^{-t / \tau_{X}} \times \Delta t .
$$

We define the energy deposition fraction at a particular redshift $z$ to be the ratio of amount of energy deposited at that redshift, $\Delta E_{\mathrm{dep}}$, to the energy injected at $t=\tau_{X}$ within a timestep, 


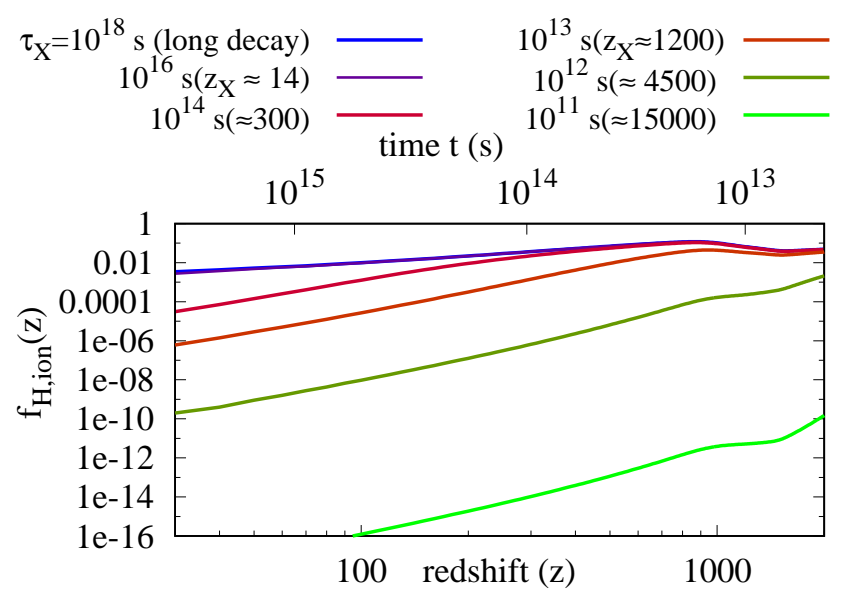

Figure 6: Fraction of energy going to direct hydrogen ionization as a function of redshift for decay to $100 \mathrm{GeV}$ electron-positron pair for different lifetimes. Note that the energy injection histories for $\tau_{X}=10^{18} \mathrm{~s}$ and $10^{16} \mathrm{~s}$ are identical.

i.e. energy deposition fraction $f_{\text {total }}(z)$ is given by,

$$
f_{\text {total }}(z)=\frac{\Delta E_{\mathrm{dep}}(z) / \Delta t}{\Delta E_{\mathrm{inj}} / \Delta t}=\frac{\Delta E_{\mathrm{dep}}(z) / \Delta t}{\frac{f_{X}}{\tau_{X}} \rho_{c} c^{2}(1+z)^{3} e^{-1.0}},
$$

and similarly for fractions of energy deposited into any particular channel such as ionization, excitation etc. We have checked that our timesteps are small enough such that the energy deposition fraction is independent of the timestep used. The dark matter annihilation module in Recfast++ $[47,48]$ uses on-the-spot energy deposition fraction as was suggested in [7]. We have modified this module to use our energy deposition fractions.

We plot the fraction of energy going into hydrogen ionization as a function of redshift with standard recombination history in Fig. 6. We neglect the fact that the recombination history is modified due to energy injection. This is a good approximation since slight modification to the recombination history with energy injection will have a negligible effect on energy deposition fraction. Significant modification to recombination history is excluded by existing cosmological data [37]. For long lifetimes $\left(\tau_{X}>>10^{13} \mathrm{~s}\right)$, energy deposition history $\left(f_{\text {total }}(z), f_{\mathrm{H} \text {,ion }}(z)\right.$ etc. $)$ becomes independent of the lifetime as shown in Fig. 6 and 7 . This can also be seen from Eq. 4.2. For $t / \tau_{X} \rightarrow 0$, the only time dependence that remains is that of dilution of dark matter energy density due to the expansion of the Universe which is independent of the lifetime of the dark matter and the only effect the lifetime $\tau_{X}$ has is to change the total amount of injected energy. For long lifetimes, Eq. 4.3 becomes,

$$
\frac{\Delta E_{\mathrm{dep}}(z)}{\Delta t}=f_{\text {total }}(z) \frac{f_{X}}{\tau_{X}} \rho_{c} c^{2}(1+z)^{3} e^{-1.0} .
$$

Since $f_{\text {total }}(z)$ is independent of $\tau_{X}$, the deposited energy is just a function of $f_{X} / \tau_{X}$, i.e. $\tau_{X}$ just affects the total energy deposited but not how that energy is deposited as a function 


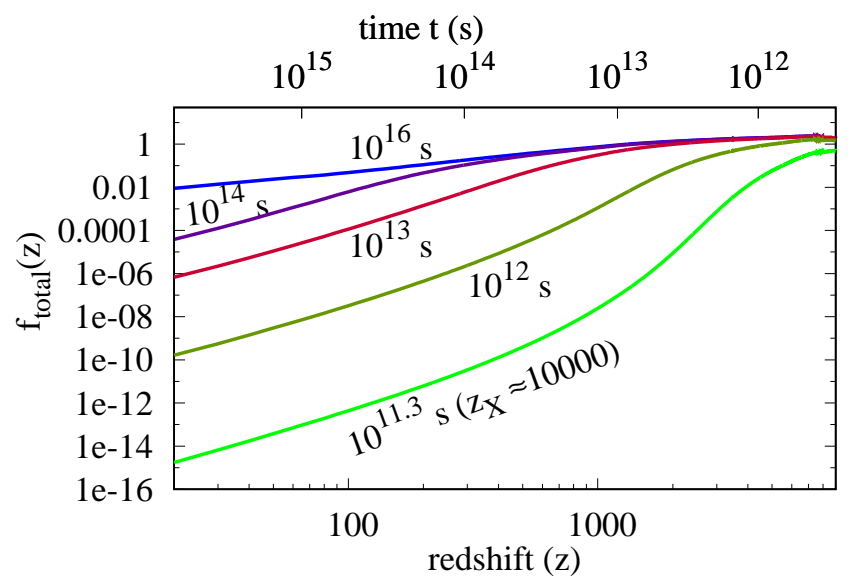

Figure 7: Total energy deposition fraction, as defined in Eq. 4.3, as a function of redshift for decay to $100 \mathrm{GeV}$ electron-positron pair for different lifetimes.

of redshift. Therefore, for $\tau_{X}>>10^{13} \mathrm{~s}$, the constraints on $f_{X}$ are defined by constant total energy injection, i.e. $\frac{f_{X}}{\tau_{X}}=$ constant or $f_{X} \propto \tau_{X}$. We thus need to derive constraint for only one $\tau_{X}$ and analytically scale the constraints for any other $\tau_{X}$ as long as the condition $\tau_{X}>>10^{13} \mathrm{~S}$ is satisfied. For shorter lifetimes, the contribution of $e^{\left(-t / \tau_{X}\right)}$ in Eq. 4.2 is important. For $\tau_{X} \lesssim 10^{13} \mathrm{~s}$, most of the energy is deposited in the ionized Universe while some photons survive carrying a small fraction of energy until recombination $(z<2000)$. Thus, the constraints become weaker again with decreasing $\tau_{X}$. Even for $z_{X}$ as high as $10000\left(\tau_{X} \approx 10^{11.3}\right)$, a tiny fraction of energy survives until recombination. This can be seen in Fig. 6 as well as in Fig. 7 . This small fraction of surviving energy is still large enough for CMB anisotropies to provide constraints competitive with the other probes.

\section{$5 \quad$ Planck CMB constraints on dark matter decay}

We consider electromagnetic energy injection from dark matter decay to monochromatic electron-positron pair or photon pair to derive constraints on fraction of decaying dark matter (compared to total dark matter) from CMB anisotropy calculations. We do Markov chain Monte Carlo (MCMC) analysis using publicly available code CosmoMC [60] in combination with Recfast ++ module of CosmoRec [47, 48] to get CMB anisotropy constraints as a function of lifetime and mass of dark matter. We simultaneously fit for $\Lambda$ CDM cosmological parameters plus $f_{X}$ using Planck 2015 PlikTT,TE,EE and lowTEB likelihood [61]. Our results are shown as $2 \sigma$ upper limits on $f_{X} f_{\mathrm{EM}}$ in Fig. 8 as a function of $\tau_{X}$, where $f_{\mathrm{EM}}$ is the fraction of decay energy going into visible electromagnetic particles and $1-f_{\mathrm{EM}}$ is the fraction lost to invisible particles such as neutrinos, dark radiation or other particles in the dark sector. CMB anisotropy constraints depend on the initial energy of injected particle or equivalently the mass of dark matter particle and varying the mass gives the band in Fig. 8. The constraints are strongest for $\tau_{X} \approx 10^{13} \mathrm{~s}\left(z_{X} \approx 1200\right)$ which is close to the peak of the CMB visibility 
function [26, 27]. For both longer and shorter lifetimes (compared to the recombination epoch), the constraints get weaker. Our calculations are in broad agreement for $\tau_{x}>10^{13} \mathrm{~s}$ with the work of [30]. For very long lifetime, the constraints follow the scaling $f_{X} \propto \tau$ as discussed in the previous section. For lifetime less than $10^{13} \mathrm{~s}$, the authors in [31] have used the energy deposition fraction of [38] to obtain constraints. However, they have assumed explicit factorization of deposition fraction to an ionization fraction and a redshift dependent part which may be invalid when ionization fraction is very high (see Fig. 8 of [62] and related discussions around Fig. 11 of [30]).

In deriving these constraints we assumed monochromatic decay of dark matter, i.e. the energy of injected photons $E_{\gamma}=m_{X} / 2$, where $m_{X}$ is the mass of the decaying dark matter particle and the kinetic energy of the injected electron-positron pairs is given by $E_{\mathrm{e}}=\left(m_{X}-\right.$ $\left.m_{\mathrm{e}}\right) / 2$. The band represents a range of energies from $10 \mathrm{keV}$ to $1 \mathrm{TeV}$ for the initial photons, electrons and positrons. Any general decay channel of an unstable particle would produce a spectrum of energies for the initial photons, electrons and positrons, once the initial unstable standard model particles have decayed. The constraints on decay into any channel would still be given by the band shown in Fig. 8, as long as most of the decay energy is in the particles with energy $\gtrsim 10 \mathrm{keV}$.

In Fig. 9, we plot the constraints from dark matter decay to electron-positron and photon pairs for a few dark matter masses individually with $f_{\mathrm{EM}}=1$ and assuming monochromatic decay channel. For $\tau_{X}>10^{13}$ s, constraints for the photon channel are slightly weaker compared to the electron-positron channel for the same dark matter mass. The high energy photons are less efficient in depositing their energies for long lifetimes. Electrons and positrons with the same energy would produce lower energy photons from ICS which are more efficient in depositing their energies to the baryonic gas. However, this difference vanishes for very high energies $(>\mathrm{GeV})$. This is to be expected as cascade evolution proceeds through cyclic production of electron, positrons and photons, making $e^{-} e^{+}$and photon pair injection indistinguishable. The high energy photons and $e^{-} e^{+}(\sim \mathrm{TeV})$ give the strongest constraints for short lifetimes $\left(\tau_{X}<10^{13} \mathrm{~s}\right)$ as the high energy gamma rays have a greater chance to survive until $z<1000$ and deposit a non-negligible fraction of their energy after recombination thus influencing the residual free electron fraction.

We find that the CMB anisotropy constraints are stronger compared to BBN and CMB spectral distortions for $z_{X} \lesssim 10000$ for all initial energies except for photon injection just above the helium photo-dissociation threshold of $\approx 20 \mathrm{MeV}$. The strongest constraints from BBN correspond to injection of photons with energy $26 \mathrm{MeV}$ and are shown in Fig. 8. In this case the BBN constraints are stronger until significantly lower redshifts $(z \sim 7000-8000)$ as explained in next section. We discuss the BBN constraints in detail in the next section.

We note that the energy injection from dark matter decay does not have significant degeneracies with other cosmological parameters [30]. In particular the best-fit/mean values of the $6 \Lambda \mathrm{CDM}$ parameters does not change significantly. We give the full parameter table as well as 2-parameter probability density function (PDF) contour plots of dark matter decay fraction vs standard $\Lambda \mathrm{CDM}$ parameters for a few illustrative cases in Appendix D. 


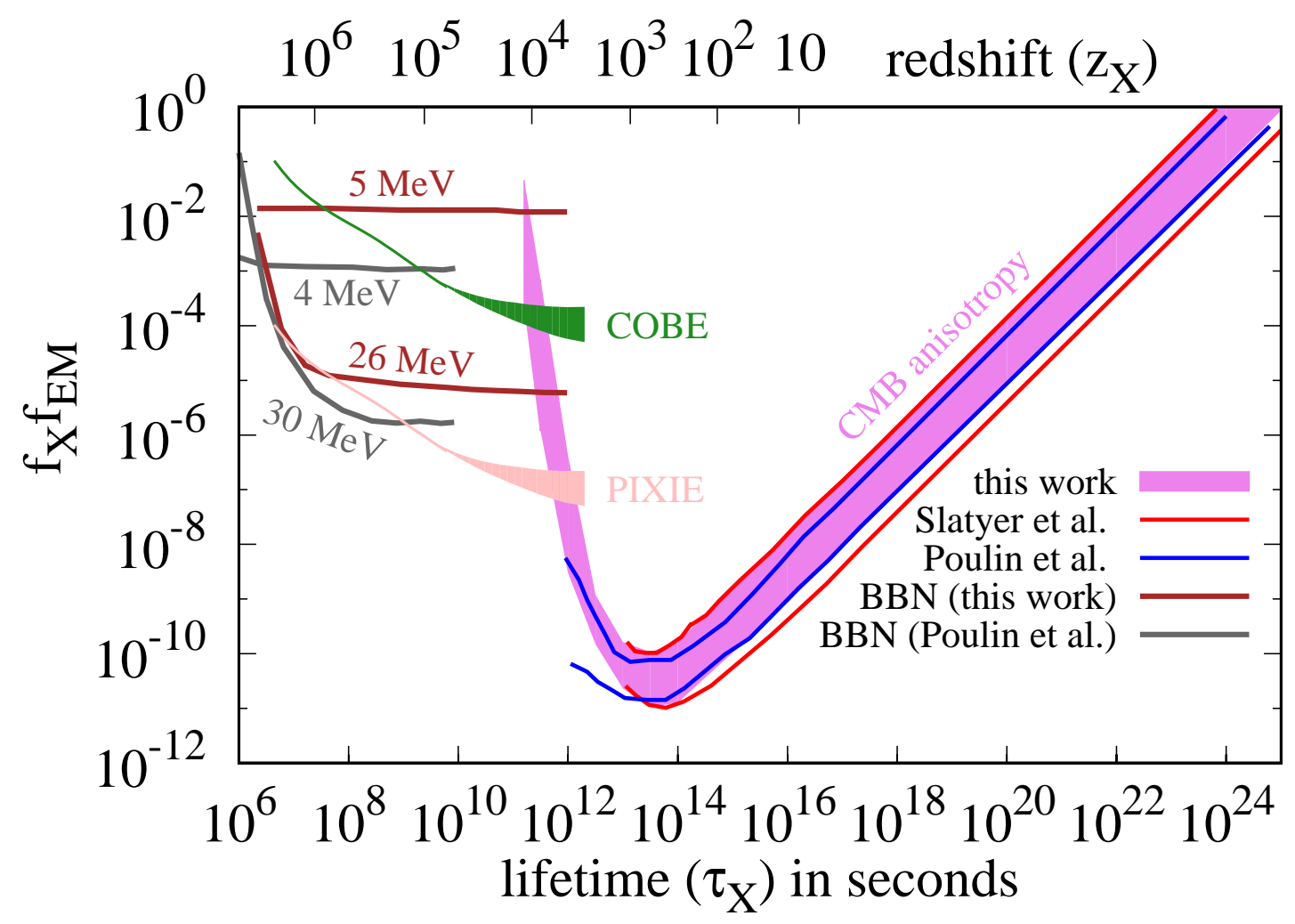

Figure 8: 2- $\sigma$ constraints on electromagnetic decay of dark matter with the energy of injected electrons, positrons and photons in the range $10 \mathrm{keV}-1 \mathrm{TeV}$. Comparison with previous calculations on CMB anisotropy ( Slatyer et al. [30], Poulin et al. [31]) is also shown. Energydependent spectral distortion constraints of [63] and extrapolated constraints for Primordial Inflation Explorer (PIXIE) [64], assuming a factor of 1000 improvement over COBE-FIRAS, are also shown. The two lines for BBN are our strongest constraints from ${ }^{4}$ He destruction (stronger) and ${ }^{2} \mathrm{H}$ destruction for photon injection with the injected photon energy of $E_{\gamma}=$ $26 \mathrm{MeV}$ and $5 \mathrm{MeV}$ respectively. For comparison we also show the constraints from Poulin et al. [43] for $E_{\gamma}=30 \mathrm{MeV}$ and $E_{\gamma}=4 \mathrm{MeV}$.

\subsection{CMB spectral distortions}

Electromagnetic energy injections at $z \lesssim 2 \times 10^{6}$ result in distortion of CMB spectrum from a Planck spectrum [65-68]. The Cosmic Background Explorer-Far Infrared Absolute Spectrophotometer (COBE-FIRAS) [69] observations of the CMB spectrum, therefore, constrain energy injection from dark matter decay to electromagnetic particles. Almost all previous calculations have assumed the distortions to be y-type $\left(z \lesssim 10^{5}\right)$ or $\mu$ type $\left(10^{5} \lesssim z \lesssim 2 \times 10^{6}\right)$ $[70,71]$. Recently, we showed that there assumptions are not strictly correct. In particular, even after taking into account intermediate or i-type distortions [72, 73], e.g. in [63, 74], there is significant corrections to non-relativistic y-type and i-type distortions [46, 63] since 


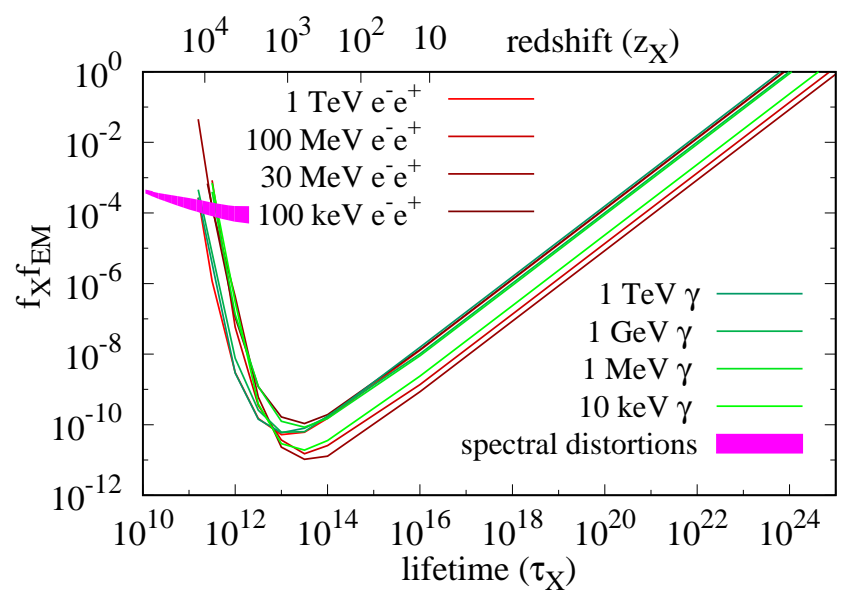

Figure 9: Constraints $(2-\sigma)$ on electromagnetic decay of dark matter for different dark matter masses (or initial energies of injected particles). Also shown are the COBE-FIRAS spectral distortion constraints of [63].

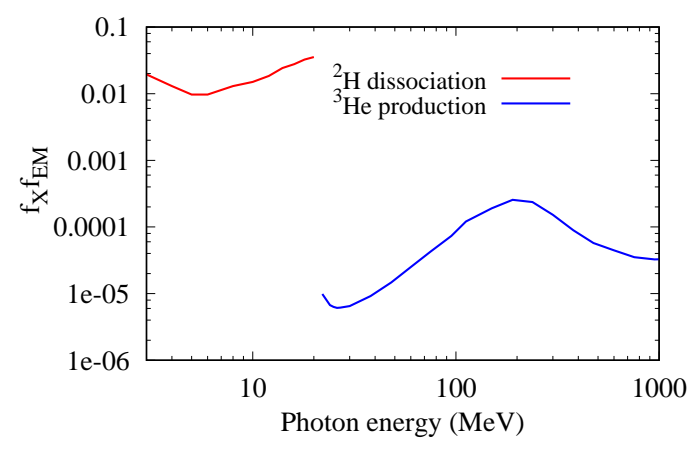

(a) BBN constraints $(2-\sigma)$ as a function of injected photon energy for $z_{X}=10000$.

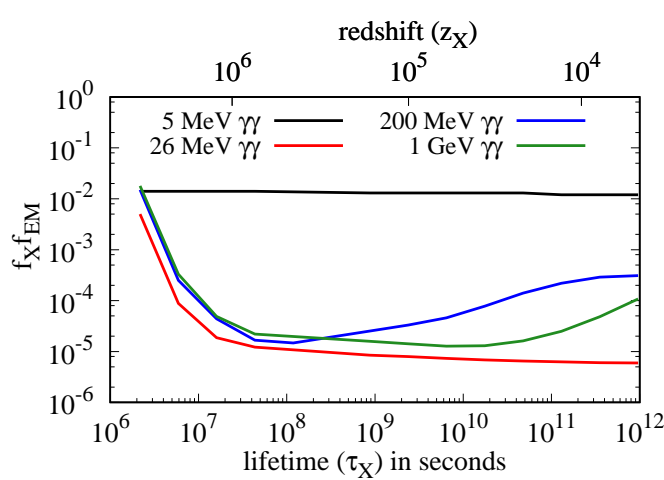

(b) BBN constraints $(2-\sigma)$ as a function of lifetime for different injected photon energies.

Figure 10: Variation of BBN constraints as a function of photon energy and lifetime.

the injected particles have energy $\gtrsim m_{\mathrm{e}}$. It was shown in [63] that, the spectral distortion constraints are energy dependent and can be relaxed with respect to the constraints obtained assuming y-type spectrum by a factor of 4 to 5 . We use the results of [63] for spectral distortions constraints in Fig. 8. The non-relativistic dark matter energy density decreases due to the expansion of the Universe as $\propto(1+z)^{3}$ while CMB energy density $\rho_{\gamma} \propto(1+z)^{4}$. The fractional injected energy density $\frac{\Delta E_{\text {inj }}}{\rho_{\gamma}} \propto \frac{1}{1+z}$ for same fraction $f_{X}$ of decaying dark matter. Therefore, for longer decay lifetimes (or lower decay redshifts) constraints on decaying dark matter get stronger in general. However, the scaling is not exactly $(1+z)$ since the electromagnetic cascade evolution also has a redshift dependence [63]. 


\begin{tabular}{l|c|} 
Reactions & photo-dissociation threshold $(\mathrm{MeV})$ \\
\hline${ }^{2} \mathrm{H}+\gamma \rightarrow \mathrm{n}+\mathrm{p}$ & 2.22 \\
${ }^{3} \mathrm{He}+\gamma \rightarrow{ }^{2} \mathrm{H}+\mathrm{p}$ & 5.49 \\
${ }^{3} \mathrm{He}+\gamma \rightarrow \mathrm{n}+\mathrm{p}+\mathrm{p}$ & 7.718 \\
${ }^{4} \mathrm{He}+\gamma \rightarrow{ }^{3} \mathrm{H}+\mathrm{p},{ }^{3} \mathrm{H} \rightarrow{ }^{3} \mathrm{He}+e^{-}+v_{e}$ & 19.81 \\
${ }^{4} \mathrm{He}+\gamma \rightarrow{ }^{3} \mathrm{He}+\mathrm{n}$ & 20.58 \\
${ }^{4} \mathrm{He}+\gamma \rightarrow{ }^{2} \mathrm{H}+{ }^{2} \mathrm{H}$ & 23.85 \\
${ }^{4} \mathrm{He}+\gamma \rightarrow{ }^{2} \mathrm{H}+\mathrm{n}+\mathrm{p}$ & 26.07
\end{tabular}

Table 1: Photo-dissociation reactions included in this paper and their threshold energy.

\begin{tabular}{l|c|r} 
Elements & theoretical value $(1 \sigma)$ & observational value $(1 \sigma)$ \\
\hline$n_{2} \mathrm{H} / n_{\mathrm{H}}$ & $(2.58 \pm 0.13) \times 10^{-5}[75]$ & $(2.53 \pm 0.04) \times 10^{-5}[75]$ \\
$Y_{p}$ & $0.24709 \pm 0.00025[75]$ & $0.2449 \pm 0.0040[76]$ \\
$n_{3} \mathrm{He} / n_{\mathrm{H}}$ & $(10.039 \pm 0.090) \times 10^{-6}[75]$ & $1.5 \times 10^{-5}$ (2o upper limit) [77]
\end{tabular}

Table 2: Theoretical predictions and observational bounds on primordial abundance of light elements. The ratio of number density of deuterium $\left(n_{2} \mathrm{H}\right)$ and helium-3 $\left(n_{3} \mathrm{He}\right)$ to hydrogen $\left(n_{\mathrm{H}}\right)$ is quoted. For Helium-4 $\left({ }^{4} \mathrm{He}\right)$ the mass fraction, $Y_{P}$, is the ratio of helium mass density to the total mass density of hydrogen and helium.

\section{$6 \quad$ BBN constraints}

High energy photons (energy greater than deuterium $\left({ }^{2} \mathrm{H}\right)$, helium-3 $\left({ }^{3} \mathrm{He}\right)$ and helium-4 $\left({ }^{4} \mathrm{He}\right)$ photo-dissociation threshold) can change the abundance of primordial ${ }^{2} \mathrm{H},{ }^{3} \mathrm{He}$ and ${ }^{4} \mathrm{He}$. Note that any ${ }^{3} \mathrm{H}$ produced from destruction of ${ }^{4} \mathrm{He}$ immediately (on time scale much shorter than the age of the Universe at recombination) decays to ${ }^{3} \mathrm{He}$. Requiring these elements to be not over- or under-produced (so that the theoretical calculations and observations are not in tension), puts constraints on the energy injection of particles with energy greater than 2.22 MeV. The photo-dissociation processes that are important for changing the primordial element abundances and included in our calculations are given in Table 1 along with the corresponding photo-dissociation thresholds. We use the fits provided in [78] for the photodissociation cross sections. Abundance of primordial elements can constrain electromagnetic energy injection after the primordial nucleosynthesis is over at $z \lesssim 10^{8}$. For a particular energy injection model, the highest redshift at which energy injection can be constrained by $\mathrm{BBN}$ depends upon the injected photon energy [43] and its pair-production threshold on $\mathrm{CMB}$ photons. Since, the CMB photons outnumber baryons by more than nine order 
of magnitude, pair production on CMB dominates every other electromagnetic and nuclear process whenever it is kinematically allowed. Pair-production on CMB photons produces $e^{-} e^{+}$ pairs which produce lower energy photons through ICS. When pair-production threshold is equal to the photo-dissociation threshold of a particular reaction at some redshift $z_{\mathrm{th}}$, all of the energy of the injected photons gets immediately degraded to low energy photons that are not able to dissociate nuclei, making BBN constraint from that particular reaction exponentially weaker for $z \gtrsim z_{\text {th }}$.

The BBN constraints on decaying dark matter have been previously studied in [41-45]. Early calculations of the effect of electromagnetic energy injection on abundances of elements assumed that any high energy particle injection results in an initial universal photon spectrum, independent of the injected particle energy. This assumption was shown to be inaccurate in $[43,45]$ for photon injection in the energy range $\sim \mathrm{MeV}-100 \mathrm{MeV}$. In particular, it was shown by [43] that the constraints for photon injection with $\sim 30 \mathrm{MeV}$ energy can be an order of magnitude stronger compared to those obtained under the assumption of universal photon spectrum [41]. However, these calculations [41-45] still make a quasi-static or on-thespot approximation to derive the BBN constraints i.e. they assume that energy deposition happens on a time scale much faster than the Hubble time.

We relax the quasi-static/one-the-spot assumption also and explicitly evolve the electromagnetic cascade in an expanding Universe with our code to obtain the BBN constraints. The theoretical predictions and observed abundances for ${ }^{2} \mathrm{H},{ }^{3} \mathrm{He}$ and ${ }^{4} \mathrm{He}$ are given in Table 2. We use the difference between the observed 2- $\sigma$ upper limit and theoretically predicted $2-\sigma$ lower limit as the maximum amount of element that can be produced by dissociation of a heavier element. Similarly, the difference between the theoretical 2- $\sigma$ upper limit and $2-\sigma$ observed lower limit gives the maximum amount of destruction allowed for an element. We don't consider ${ }^{3} \mathrm{He}$ destruction as there is only an upper limit on its abundance. Since the abundance of deuterium is small compared to the background electrons and ions by a factor of $\sim 10^{5},{ }^{2} \mathrm{H}$ is an unlikely target for an energetic photon making the constraint in this case very weak. The strongest constraints come from destruction of ${ }^{4} \mathrm{He}$ since it has a much larger abundance, $\approx 8 \%$ of hydrogen by number and the deuterium destruction constraints are relevant only below the ${ }^{4} \mathrm{He}$ destruction threshold, $E_{\gamma}<19.81 \mathrm{MeV}$. The photons with energy $\gtrsim 19.81 \mathrm{MeV}$ photo-dissociate ${ }^{4} \mathrm{He}$ to ${ }^{3} \mathrm{He},{ }^{3} \mathrm{H}$ giving strong constraints due to ${ }^{3} \mathrm{He}$ over-production.

We show, in Figs. 10a and 10b, the BBN constraints from dark matter decay into photons for $z_{X}=10000$, as a function of photon energy. We see that the constraints depend very strongly on the energy of injected particles and can vary by more than an order of magnitude. The constraints are the strongest at $E_{\gamma} \approx 26 \mathrm{MeV}$ and $5 \mathrm{MeV}$ for ${ }^{4} \mathrm{He}$ and ${ }^{2} \mathrm{H}$ destruction respectively and these strongest limits are shown in Fig. 8. We can interpret these constraints for a general decay channel after defining $f_{\mathrm{EM}}$ to be approximately the energy injected in electromagnetic particles above the respective thresholds of $2.22 \mathrm{MeV}$ and $19.81 \mathrm{MeV}$. The $5 \mathrm{MeV}$ photon is below the threshold of ${ }^{4} \mathrm{He}$ photo-dissociation and therefore these photons can only destroy ${ }^{2} \mathrm{H}$. We do not show the constraints from ${ }^{2} \mathrm{H}$ over-production 
since the cross-section for ${ }^{4} \mathrm{He}$ destruction to ${ }^{3} \mathrm{He}$ is an order of magnitude larger compared to the photo-dissociation of ${ }^{4} \mathrm{He}$ to ${ }^{2} \mathrm{H}$ and thus the latter gives weaker constraints. Since the rates for all relevant processes which compete with photo-dissociation depend on the number density of targets (i.e. background electrons, ions and photons) and thus have the same redshift dependence $\left(\propto(1+z)^{3}\right)$, the constraints are independent of dark matter lifetime for $\tau_{X} \gtrsim 10^{8}$ s. However, for $\tau_{X} \lesssim 10^{8} \mathrm{~s}$, the threshold energy for pair production on CMB photons becomes similar to the photo-dissociation threshold and the constraints become sensitive to $\tau_{X}$

We also show comparison with the published results from [43] for $E_{\gamma}=4 \mathrm{MeV}$ and $30 \mathrm{MeV}$. Our constraints are weaker by a factor of 3 for ${ }^{3} \mathrm{He}$ overproduction and almost an order of magnitude for ${ }^{2} \mathrm{H}$ destruction. This is expected, since we evolve the electromagnetic cascades in the expanding Universe and allow the energy to be deposited not instantaneously but in a delayed manner. There is thus a higher probability for the energy to be lost to other processes such as heating, decreasing the fraction of energy going into the destruction of primordial elements.

\section{Conclusions}

In this work, we obtain constraints on decaying dark matter from Planck observations of CMB temperature and polarization anisotropy power spectra and abundances of light elements. Our CMB anisotropy results broadly agree with the previous calculations for dark matter lifetime $\tau_{X} \gtrsim 10^{13} \mathrm{~s}$. We give the first CMB anisotropies constraints for $\tau_{X}<10^{13}$ s upto the point where the constraints from $\mathrm{CMB}$ spectral distortions and $\mathrm{BBN}$ are stronger. We find that for general energies, the CMB anisotropies are a powerful probe of energy injection for redshifts as high as 10000, providing the strongest constraints today and thereby excluding a new region of parameter space. For these calculations, we have developed a new code which evolves high energy particle cascades taking into account background evolution of ionization fraction of hydrogen, helium, singly ionized helium and background expansion. We do not rely on factorization of the full calculation into low and high energy part and evolve both sub-keV and higher energy particles consistently in one unified code. For completeness, we also show the CMB spectral distortion constraints from COBE-FIRAS data and give forecasts for a future PIXIE like mission. We also calculate primordial elements abundance constraints with the same code and find that taking delayed deposition of energy is important for accurate constraints. Taking the delayed deposition of energy into account weakens the constraints considerably compared to the instantaneous deposition approximation made in previous calculations.

Even though, we have only considered monochromatic electron-positron and photon pair injections, it is straightforward to interpret our constraints for any general decay channel. Our calculations can also be generalized to other energy injection processes with arbitrary spectrum, which we leave for future work. One such example would be evaporation of primordial black holes. Our calculations suggest that the CMB anisotropies can constrain black 
hole evaporation at higher redshifts or lower black hole masses than what has been considered in the literature so far.

Although the CMB anisotropy analysis only provides constraints on amount of energy injection, our calculations can be extended to a more direct probe for electromagnetic energy injection around or before recombination, namely the cosmological recombination spectrum. Since, recombination is a non-equilibrium process, a characteristic distortion signal with information about recombination as well as background CMB spectrum gets imprinted on the initially Planckian CMB spectrum. Energy injection around recombination modifies this signal in an unique way [57]. We have argued that the excitations to higher atomic energy levels will be important for accurate predictions of recombination line spectral distortions. We however leave a detailed study for future work.

\section{Acknowledgements}

We acknowledge the use of computational facilities of Department of Theoretical Physics at Tata Institute of Fundamental Research, Mumbai. This work was supported by Max Planck Partner Group for cosmology of Max Planck Institute for Astrophysics Garching at Tata Institute of Fundamental Research funded by Max-Planck-Gesellschaft. This work was also supported by Science and Engineering Research Board (SERB) of Department of Science and Technology, Government of India grant no. ECR/2015/000078. We acknowledge support of the Department of Atomic Energy, Government of India, under project no. 12-R\&D-TFR$5.02-0200$.

\section{References}

[1] N. Aghanim et al. Planck 2018 results. VI. Cosmological parameters. ArXiv e-prints, July 2018. arXiv: 1807.06209, [ADS].

[2] David N. Spergel and Paul J. Steinhardt. Observational Evidence for Self-Interacting Cold Dark Matter. Phys.Rev.Lett, 84(17):3760-3763, Apr 2000. arXiv:astro-ph/9909386, [DOI], [ADS].

[3] Torsten Bringmann, Felix Kahlhoefer, Kai Schmidt-Hoberg, and Parampreet Walia. Strong Constraints on Self-Interacting Dark Matter with Light Mediators. Phys.Rev.Lett, 118(14):141802, Apr 2017. arXiv:1612.00845, [DOI], [ADS].

[4] Manuel A. Buen-Abad, Gustavo Marques-Tavares, and Martin Schmaltz. Non-Abelian dark matter and dark radiation. Phys.Rev.D, 92(2):023531, Jul 2015. arXiv:1505.03542, [DOI], [ADS].

[5] Julien Lesgourgues, Gustavo Marques-Tavares, and Martin Schmaltz. Evidence for dark matter interactions in cosmological precision data? JCAP, 2016(2):037, Feb 2016. arXiv:1507.04351, [DOI], [ADS].

[6] J. A. Adams, S. Sarkar, and D. W. Sciama. Cosmic microwave background anisotropy in the decaying neutrino cosmology. MNRAS, 301:210-214, November 1998.

arXiv: astro-ph/9805108, [DOI], [ADS]. 
[7] X. Chen and M. Kamionkowski. Particle decays during the cosmic dark ages. Phys.Rev.D, 70(4):043502, August 2004. arXiv:astro-ph/0310473, [DOI], [ADS].

[8] S. W. Hawking. Particle creation by black holes. Communications in Mathematical Physics, 43:199-220, August 1975. [DOI], [ADS].

[9] Patrick Stöcker, Michael Krämer, Julien Lesgourgues, and Vivian Poulin. Exotic energy injection with ExoCLASS: application to the Higgs portal model and evaporating black holes. JCAP, 2018(3):018, Mar 2018. arXiv:1801.01871, [DOI], [ADS].

[10] T. R. Slatyer, N. Padmanabhan, and D. P. Finkbeiner. CMB constraints on WIMP annihilation: Energy absorption during the recombination epoch. Phys.Rev.D, 80(4):043526, August 2009. arXiv:0906.1197, [DOI], [ADS].

[11] G. Hütsi, J. Chluba, A. Hektor, and M. Raidal. WMAP7 and future CMB constraints on annihilating dark matter: implications for GeV-scale WIMPs. AËA, 535:A26, Nov 2011. arXiv:1103.2766, [DOI], [ADS].

[12] Tracy R. Slatyer. Indirect dark matter signatures in the cosmic dark ages. I. Generalizing the bound on s-wave dark matter annihilation from Planck results. Phys.Rev.D, 93(2):023527, Jan 2016. arXiv:1506.03811, [DOI], [ADS].

[13] Cora Dvorkin, Kfir Blum, and Marc Kamionkowski. Constraining dark matter-baryon scattering with linear cosmology. Phys.Rev.D, 89(2):023519, Jan 2014. arXiv:1311.2937, [DOI], [ADS].

[14] Benjamin Audren, Julien Lesgourgues, Gianpiero Mangano, Pasquale Dario Serpico, and Thomas Tram. Strongest model-independent bound on the lifetime of Dark Matter. JCAP, 2014(12):028, Dec 2014. arXiv:1407.2418, [DOI], [ADS].

[15] S. Weinberg. Cosmological constraints on the scale of supersymmetry breaking. Physical Review Letters, 48:1303-1306, May 1982. [DOI], [ADS].

[16] H. Goldberg. Constraint on the photino mass from cosmology. Physical Review Letters, 50:1419-1422, May 1983. [DOI], [ADS].

[17] T. Moroi, H. Murayama, and M. Yamaguchi. Cosmological constraints on the light stable gravitino. Physics Letters B, 303:289-294, April 1993. [DOI], [ADS].

[18] Jonathan L. Feng, Arvind Rajaraman, and Fumihiro Takayama. Superweakly Interacting Massive Particles. Phys.Rev.Lett, 91:011302, Jul 2003. arXiv:hep-ph/0302215, [DOI], [ADS].

[19] Jonathan L. Feng, Arvind Rajaraman, and Fumihiro Takayama. Superweakly interacting massive particle dark matter signals from the early Universe. Phys.Rev.D, 68:063504, Sep 2003. arXiv:hep-ph/0306024, [DOI], [ADS].

[20] Hsin-Chia Cheng, Jonathan L. Feng, and Konstantin T. Matchev. Kaluza-Klein Dark Matter. Phys.Rev.Lett, 89:211301, Oct 2002. arXiv:hep-ph/0207125, [DOI], [ADS].

[21] Nima Arkani-Hamed, Douglas P. Finkbeiner, Tracy R. Slatyer, and Neal Weiner. A theory of dark matter. Phys.Rev.D, 79(1):015014, Jan 2009. arXiv:0810.0713, [DOI], [ADS].

[22] Torsten Bringmann and Christoph Weniger. Gamma ray signals from dark matter: Concepts, status and prospects. Physics of the Dark Universe, 1(1):194 - 217, 2012. Next Decade in Dark Matter and Dark Energy. URL:

http://www.sciencedirect.com/science/article/pii/S221268641200009X, [DOI]. 
[23] Jonathan L. Feng. Dark Matter Candidates from Particle Physics and Methods of Detection. Annual Review of Astronomy and Astrophysics, 48:495-545, Sep 2010. arXiv:1003.0904, [DOI], [ADS].

[24] Gianfranco Bertone, Dan Hooper, and Joseph Silk. Particle dark matter: evidence, candidates and constraints. Physics Reports, 405(5-6):279-390, Jan 2005. arXiv:hep-ph/0404175, [DOI], [ADS].

[25] S. Galli, T. R. Slatyer, M. Valdes, and F. Iocco. Systematic uncertainties in constraining dark matter annihilation from the cosmic microwave background. Phys.Rev.D, 88(6):063502, September 2013. arXiv:1306.0563, [DOI], [ADS].

[26] Y. B. Zeldovich, V. G. Kurt, and R. A. Sunyaev. Recombination of Hydrogen in the Hot Model of the Universe. Soviet Journal of Experimental and Theoretical Physics, 28:146, January 1969. [ADS].

[27] P. J. E. Peebles. Recombination of the Primeval Plasma. ApJ, 153:1, July 1968. [DOI], [ADS].

[28] N. Padmanabhan and D. P. Finkbeiner. Detecting dark matter annihilation with CMB polarization: Signatures and experimental prospects. Phys.Rev.D, 72(2):023508, July 2005. arXiv: astro-ph/0503486, [DOI], [ADS].

[29] S. Galli, F. Iocco, G. Bertone, and A. Melchiorri. CMB constraints on dark matter models with large annihilation cross section. Phys.Rev.D, 80(2):023505, July 2009. arXiv:0905.0003, [DOI], [ADS].

[30] Tracy R. Slatyer and Chih-Liang Wu. General constraints on dark matter decay from the cosmic microwave background. Phys.Rev.D, 95(2):023010, Jan 2017. arXiv:1610.06933, [DOI], [ADS].

[31] Vivian Poulin, Julien Lesgourgues, and Pasquale D. Serpico. Cosmological constraints on exotic injection of electromagnetic energy. JCAP, 2017(3):043, Mar 2017. arXiv:1610.10051, [DOI], [ADS].

[32] J. M. Shull and M. E. van Steenberg. X-ray secondary heating and ionization in quasar emission-line clouds. ApJ, 298:268-274, November 1985. [DOI], [ADS].

[33] Steven R. Furlanetto and Samuel Johnson Stoever. Secondary ionization and heating by fast electrons. MNRAS, 404(4):1869-1878, Jun 2010. arXiv:0910.4410, [DOI], [ADS].

[34] M. Valdés, C. Evoli, and A. Ferrara. Particle energy cascade in the intergalactic medium. MNRAS, 404(3):1569-1582, May 2010. arXiv:0911.1125, [DOI], [ADS].

[35] Toru Kanzaki and Masahiro Kawasaki. Electron and photon energy deposition in the Universe. Phys.Rev.D, 78(10):103004, Nov 2008. arXiv:0805.3969, [DOI], [ADS].

[36] T. Kanzaki, M. Kawasaki, and K. Nakayama. Effects of Dark Matter Annihilation on the Cosmic Microwave Background. Progress of Theoretical Physics, 123(5):853-865, May 2010. arXiv:0907.3985, [DOI], [ADS].

[37] Planck Collaboration and P. A. R. Ade. Planck 2015 results. XIII. Cosmological parameters. AEA, 594:A13, Sep 2016. arXiv: 1502.01589, [DOI], [ADS].

[38] Tracy R. Slatyer. Indirect dark matter signatures in the cosmic dark ages. II. Ionization, 
heating, and photon production from arbitrary energy injections. Phys.Rev.D, 93(2):023521, Jan 2016. arXiv:1506.03812, [DOI], [ADS].

[39] J. Ellis, D. V. Nanopoulos, and S. Sarkar. The cosmology of decaying gravitinos. Nuclear Physics B, 259:175-188, September 1985. [DOI], [ADS].

[40] J. Ellis, G. B. Gelmini, J. L. Lopez, D. V. Nanopoulos, and S. Sarkar. Astrophysical constraints on massive unstable neutral relic particles. Nuclear Physics B, 373:399-437, April 1992. [DOI], [ADS].

[41] M. Kawasaki and T. Moroi. Electromagnetic Cascade in the Early Universe and Its Application to the Big Bang Nucleosynthesis. ApJ, 452:506, Oct 1995. arXiv:astro-ph/9412055, [DOI], [ADS].

[42] Masahiro Kawasaki, Kazunori Kohri, Takeo Moroi, and Yoshitaro Takaesu. Revisiting big-bang nucleosynthesis constraints on long-lived decaying particles. Phys.Rev.D, 97(2):023502, Jan 2018. arXiv:1709.01211, [DOI], [ADS].

[43] Vivian Poulin and Pasquale Dario Serpico. Nonuniversal BBN bounds on electromagnetically decaying particles. Phys.Rev.D, 91(10):103007, May 2015. arXiv:1503.04852, [DOI], [ADS].

[44] Marco Hufnagel, Kai Schmidt-Hoberg, and Sebastian Wild. BBN constraints on MeV-scale dark sectors. Part II: Electromagnetic decays. JCAP, 2018(11):032, Nov 2018. arXiv: 1808.09324, [DOI], [ADS].

[45] Lindsay Forestell, David E. Morrissey, and Graham White. Limits from BBN on light electromagnetic decays. Journal of High Energy Physics, 2019(1):74, Jan 2019. arXiv:1809.01179, [DOI], [ADS].

[46] Sandeep Kumar Acharya and Rishi Khatri. Rich structure of nonthermal relativistic CMB spectral distortions from high energy particle cascades at redshifts $z \lesssim 2 \times 10^{5}$. Phys.Rev.D, 99(4):043520, Feb 2019. arXiv: 1808.02897, [DOI], [ADS].

[47] J. Chluba and R. M. Thomas. Towards a complete treatment of the cosmological recombination problem. MNRAS, 412:748-764, April 2011. arXiv:1010.3631, [DOI], [ADS].

[48] S. Seager, D. D. Sasselov, and D. Scott. A New Calculation of the Recombination Epoch. ApJL, 523:L1-L5, September 1999. arXiv:astro-ph/9909275, [DOI], [ADS].

[49] Sara Seager, Dimitar D. Sasselov, and Douglas Scott. How Exactly Did the Universe Become Neutral? ApJS, 128(2):407-430, Jun 2000. arXiv:astro-ph/9912182, [DOI], [ADS].

[50] J. Chluba and R. A. Sunyaev. Induced two-photon decay of the $2 \mathrm{~s}$ level and the rate of cosmological hydrogen recombination. A\& $A$, 446:39-42, January 2006. arXiv:astro-ph/0508144, [DOI], [ADS].

[51] Eric R. Switzer and Christopher M. Hirata. Primordial helium recombination. I. Feedback, line transfer, and continuum opacity. Phys.Rev.D, 77(8):083006, Apr 2008. arXiv:astro-ph/0702143, [DOI], [ADS].

[52] J. A. Rubiño-Martín, J. Chluba, and R. A. Sunyaev. Lines in the cosmic microwave background spectrum from the epoch of cosmological helium recombination. A\&A, 485(2):377-393, Jul 2008. arXiv:0711.0594, [DOI], [ADS]. 
[53] Daniel Grin and Christopher M. Hirata. Cosmological hydrogen recombination: The effect of extremely high-n states. Phys.Rev.D, 81(8):083005, Apr 2010. arXiv:0911.1359, [DOI], [ADS].

[54] J. Chluba, G. M. Vasil, and L. J. Dursi. Recombinations to the Rydberg states of hydrogen and their effect during the cosmological recombination epoch. MNRAS, 407(1):599-612, Sep 2010. arXiv: 1003.4928, [DOI], [ADS].

[55] Yacine Ali-Haïmoud and Christopher M. Hirata. Ultrafast effective multilevel atom method for primordial hydrogen recombination. Phys.Rev.D, 82(6):063521, Sep 2010. arXiv:1006.1355, [DOI], [ADS].

[56] Y. Ali-Haïmoud and C. M. Hirata. HyRec: A fast and highly accurate primordial hydrogen and helium recombination code. Phys.Rev.D, 83(4):043513, February 2011. arXiv:1011.3758, [DOI], [ADS].

[57] J. Chluba. Could the cosmological recombination spectrum help us understand annihilating dark matter? MNRAS, 402(2):1195-1207, Feb 2010. arXiv:0910.3663, [DOI], [ADS].

[58] Hongwan Liu, Gregory W. Ridgway, and Tracy R. Slatyer. DarkHistory: A code package for calculating modified cosmic ionization and thermal histories with dark matter and other exotic energy injections. arXiv e-prints, page arXiv:1904.09296, Apr 2019. arXiv:1904.09296, [ADS].

[59] Jens Chluba and Yacine Ali-Haïmoud. COSMOSPEC: fast and detailed computation of the cosmological recombination radiation from hydrogen and helium. MNRAS, 456(4):3494-3508, Mar 2016. arXiv: 1510.03877, [DOI], [ADS].

[60] Antony Lewis and Sarah Bridle. Cosmological parameters from CMB and other data: A Monte Carlo approach. Phys.Rev.D, 66(10):103511, Nov 2002. arXiv: astro-ph/0205436, [DOI], [ADS].

[61] Aghanim et. al. Planck Collaboration. Planck 2015 results. XI. CMB power spectra, likelihoods, and robustness of parameters. A\&A, 594:A11, Sep 2016. arXiv:1507.02704, [DOI], [ADS].

[62] Tracy R. Slatyer. Energy injection and absorption in the cosmic dark ages. Phys.Rev.D, 87(12):123513, Jun 2013. arXiv:1211.0283, [DOI], [ADS].

[63] Sandeep Kumar Acharya and Rishi Khatri. New CMB spectral distortion constraints on decaying dark matter with full evolution of electromagnetic cascades before recombination. Physical Review D, 99(12):123510, Jun 2019. arXiv:1903.04503, [DOI], [ADS].

[64] A. Kogut, D. J. Fixsen, D. T. Chuss, J. Dotson, E. Dwek, M. Halpern, G. F. Hinshaw, S. M. Meyer, S. H. Moseley, M. D. Seiffert, D. N. Spergel, and E. J. Wollack. The Primordial Inflation Explorer (PIXIE): a nulling polarimeter for cosmic microwave background observations. JCAP, 2011(7):025, Jul 2011. arXiv:1105.2044, [DOI], [ADS].

[65] R. A. Sunyaev and Ya. B. Zeldovich. The interaction of matter and radiation in the hot model of the Universe, II. ApSS, 7(1):20-30, Apr 1970. [DOI], [ADS].

[66] L. Danese and G. de Zotti. Double Compton process and the spectrum of the microwave background. AESA, 107(1):39-42, Mar 1982. [ADS].

[67] Rishi Khatri and Rashid A. Sunyaev. Creation of the CMB spectrum: precise analytic solutions for the blackbody photosphere. JCAP, 2012(6):038, Jun 2012. arXiv:1203.2601, [DOI], [ADS]. 
[68] J. Chluba and R. A. Sunyaev. The evolution of CMB spectral distortions in the early Universe. MNRAS, 419(2):1294-1314, Jan 2012. arXiv:1109.6552, [DOI], [ADS].

[69] J. C. Mather, E. S. Cheng, D. A. Cottingham, R. E. Eplee, Jr., D. J. Fixsen, T. Hewagama, R. B. Isaacman, K. A. Jensen, S. S. Meyer, P. D. Noerdlinger, S. M. Read, L. P. Rosen, R. A. Shafer, E. L. Wright, C. L. Bennett, N. W. Boggess, M. G. Hauser, T. Kelsall, S. H. Moseley, Jr., R. F. Silverberg, G. F. Smoot, R. Weiss, and D. T. Wilkinson. Measurement of the cosmic microwave background spectrum by the COBE FIRAS instrument. ApJ, 420:439-444, January 1994. [DOI], [ADS].

[70] Y. B. Zeldovich and R. A. Sunyaev. The Interaction of Matter and Radiation in a Hot-Model Universe. ApSS, 4:301-316, July 1969. [DOI], [ADS].

[71] A. F. Illarionov and R. A. Siuniaev. Comptonization, the background-radiation spectrum, and the thermal history of the universe. Soviet Astronomy, 18:691-699, June 1975. [ADS].

[72] R. Khatri and R. A. Sunyaev. Beyond y and $\mu$ : the shape of the CMB spectral distortions in the intermediate epoch, $1.5 \times 10^{4} \lesssim \mathrm{z} \lesssim 2 \times 10^{5}$. JCAP, 9:016, September 2012. arXiv:1207.6654, [DOI], [ADS].

[73] J. Chluba. Green's function of the cosmological thermalization problem. MNRAS, 434:352-357, September 2013. arXiv:1304.6120, [DOI], [ADS].

[74] Matteo Lucca, Nils Schöneberg, Deanna C. Hooper, Julien Lesgourgues, and Jens Chluba. The synergy between CMB spectral distortions and anisotropies. arXiv e-prints, page arXiv:1910.04619, Oct 2019. arXiv:1910.04619, [ADS].

[75] Richard H. Cyburt, Brian D. Fields, Keith A. Olive, and Tsung-Han Yeh. Big bang nucleosynthesis: Present status. Reviews of Modern Physics, 88(1):015004, Jan 2016. arXiv:1505.01076, [DOI], [ADS].

[76] Erik Aver, Keith A. Olive, and Evan D. Skillman. The effects of He I $\lambda 10830$ on helium abundance determinations. Journal of Cosmology and Astro-Particle Physics, 2015(7):011, Jul 2015. arXiv:1503.08146, [DOI], [ADS].

[77] T. M. Bania, Robert T. Rood, and Dana S. Balser. The cosmological density of baryons from observations of $3 \mathrm{He}+$ in the Milky Way. Nature, 415, 2002. [DOI].

[78] Richard H. Cyburt, John Ellis, Brian D. Fields, and Keith A. Olive. Updated nucleosynthesis constraints on unstable relic particles. Physical Review D, 67(10):103521, May 2003. arXiv:astro-ph/0211258, [DOI], [ADS].

[79] M. Inokuti. Inelastic Collisions of Fast Charged Particles with Atoms and Molecules - The Bethe Theory Revisited. Reviews of Modern Physics, 43:297-347, July 1971. [DOI], [ADS].

[80] M. Inokuti, Y. Itikawa, and J. E. Turner. Addenda: Inelastic collisions of fast charged particles with atoms and molecules - The Bethe theory revisited. Reviews of Modern Physics, 50:23-35, January 1978. [DOI], [ADS].

[81] P. M. Stone, Y. K. Kim, and J. P. Desclaux. Electron-Impact Cross Sections for Dipole- and Spin-Allowed Excitations of Hydrogen, Helium, and Lithium. Journal of research of the National Institute of Standards and Technology, 107(4), 32733\%. doi:10.6028/jres.107.026, 2002.

[82] M. Arnaud and R. Rothenflug. An updated evaluation of recombination and ionization rates. A\&\&A Supp., 60:425-457, June 1985. [ADS]. 
[83] A. A. Zdziarski and R. Svensson. Absorption of X-rays and gamma rays at cosmological distances. ApJ, 344:551-566, September 1989. [DOI], [ADS].

[84] Planck Collaboration. Planck 2015 results. XIII. Cosmological parameters. AËA, 594:A13, Sep 2016. arXiv:1502.01589, [DOI], [ADS].

\section{A Collision cross-sections and energy loss rates for electrons and positrons}

The cross-sections and energy-loss rates for electron and positron except for excitation and ionization of neutral hydrogen and helium are given with references in [46]. We give below the cross sections for the additional atomic processes included in the present paper.

\section{A.1 Collisional excitation of neutral Hydrogen and Helium}

For collisional excitation of neutral hydrogen, we use the tabulated cross-section in CCC data base $^{2}$ for incident electron with kinetic energy from threshold to $\sim \mathrm{keV}$ energy. For higher energy, we use Bethe approximation [35, 79, 80],

$$
\begin{aligned}
\sigma(E)_{2 p} & =\frac{4 \pi a_{0}^{2}}{(E / 13.6)}\left[0.55 \times \ln \left(\frac{4 C_{2 p} E}{13.6}\right)+\frac{0.21}{(E / 13.6)}\right], \\
\sigma(E)_{2 s} & =\frac{4 \pi a_{0}^{2}}{(E / 13.6)}\left[0.12+\frac{-0.31}{(E / 13.6)}\right], \\
\sigma(E)_{n=3} & =\frac{4 \pi a_{0}^{2}}{(E / 13.6)}\left[8.9 \times 10^{-2} \ln \left(\frac{4 C_{3} E}{13.6}\right)\right],
\end{aligned}
$$

where $a_{0}$ is the Bohr radius, $\ln C_{2 p}=-0.9$, and $\ln C_{3}=-0.27$. We have only considered $3 p$ level with above cross-section. Cross-section for s level is typically one order of magnitude less compared to the $\mathrm{p}$ level.

For neutral helium excitation to $2 p$ level, we use the data and fit provided in [81],

$$
\sigma_{e H e}=\frac{4 \pi a_{0}^{2} R}{T+B+E}\left[a \ln (T / R)+b+c \frac{R}{T}\right],
$$

where $R=$ Rydberg energy, $T=$ kinetic energy of electron, $E=$ excitation energy, $B=$ binding energy of the electron to be excited, $a=0.17, b=-0.08$, and $c=0.035$.

\section{A.2 Collisional ionization of neutral Hydrogen and Helium}

For collisional ionization of neutral hydrogen at low energy, we use the cross-section tabulated in CCC database. For high energy, we use bethe approximation [35, 79, 80],

$$
\sigma(E)=\frac{4 \pi a_{0}^{2}}{E / 13.6}\left[0.28 \times \ln \left(\frac{4 C_{i} E}{13.6}\right)+\frac{\gamma_{i}}{(E / 13.6)}\right],
$$

\footnotetext{
${ }^{2}$ http://atom. curtin.edu.au/CCC-WWW/
} 
where $\ln C_{i}=3.048, \gamma_{i}=-1.63+\ln (13.6 / E)$. The spectrum of secondary electrons is given by

$$
\frac{d \sigma(E, \epsilon)}{d \epsilon}=\frac{A(E)}{1+\left(\epsilon / \epsilon_{0}\right)^{2}}
$$

with $0 \leqslant \epsilon \leqslant 0.5 \times(E-I)$, where $I$ is the ionization threshold, $\epsilon_{0}=8 \mathrm{eV}, A(E)=\frac{\sigma(E)}{\epsilon_{0}}\left[\tan ^{-1}(X(E))\right]^{-1}$ with $X(E)=\frac{E-I}{2 \epsilon_{0}}$. For neutral helium cross-section we use the fit [82], [10],

$$
\sigma(E)=10^{-14} \mathrm{~cm}^{2} \frac{1}{u(I / e V)^{2}}\left[A\left(1-\frac{1}{u}\right)+B\left(1-\frac{1}{u}\right)^{2}+C \ln u+\frac{D \ln u}{u}\right],
$$

where $u=E / I, A=17.8, B=-11, C=7, D=-23.2$, and $I=24.6 \mathrm{eV}$. The spectrum of secondary electrons is same as that of hydrogen with $\epsilon_{0}=15.8 \mathrm{eV}$ [33].

We neglect collisional excitation and ionization of singly ionized helium as during its recombination epoch $(z \sim 6000)$, the Universe is highly ionized and sub-keV electrons deposit most of their energy as heat.

\section{B Photo-ionization}

The photo-ionization cross-section used in this work is given by [10, 83],

$$
\sigma=\frac{2^{9} \pi^{2} r_{0}^{2}}{3 \alpha^{3}}\left(\frac{E_{\mathrm{thres}}}{E}\right)^{4} \frac{\exp (-4 \eta \arctan (1 / \eta))}{1-\exp (-2 \pi \eta)}
$$

where $\eta=\frac{1}{\left.\left(\frac{E}{E_{\text {thres }}}\right)-1\right)^{1 / 2}}, r_{0}$ is the electron radius, $E_{\text {thres }}=13.6 \mathrm{eV}$ for hydrogen and $54.4 \mathrm{eV}$ for singly ionized helium, and $\alpha$ is the fine structure constant. Photo-ionization cross-section of neutral helium is given by,

$$
\sigma_{\mathrm{He}}=-12 \sigma_{H}+5.1 \times 10^{-20} \mathrm{~cm}^{2}\left(\frac{E}{250 e V}\right)^{-2.65}
$$

for $50 \mathrm{eV}<\mathrm{E}<250 \mathrm{eV}$ and

$$
\sigma_{\mathrm{He}}=-12 \sigma_{H}+5.1 \times 10^{-20} \mathrm{~cm}^{2}\left(\frac{E}{250 e V}\right)^{-3.3}
$$

for $\mathrm{E}>250 \mathrm{eV}$. For other processes involving high energy photons, we refer the reader to [46].

\section{Effect of dark matter decay on the recombnation history and CMB anisotropies}

In this section, we show for completeness, the change in recombination histories for dark matter decaying during recombination $\left(\tau_{X}=10^{13} \mathrm{~s}\right)$ and before recombination $\left(\tau_{X}=10^{12} \mathrm{~s}\right)$ in Fig. 11. We also give the corresponding change in the CMB temperature and polarization power spectrum in Fig. 12. The fractional change in the temperature power spectrum for 


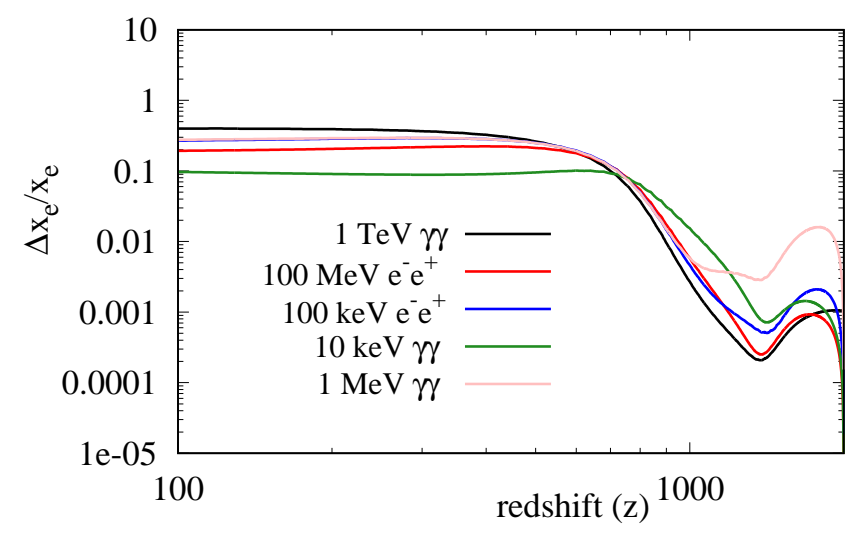

(a) $\tau_{X}=10^{12} \mathrm{~s}$

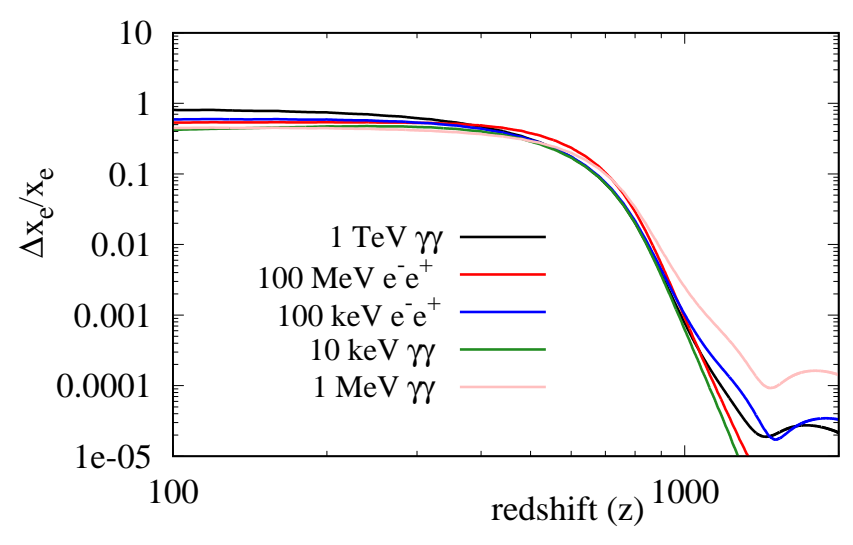

(b) $\tau_{X}=10^{13} \mathrm{~s}$

Figure 11: Change in recombination history for different dark matter decay scenarios

post recombination decay is plotted in Fig. 13, see section 3.1 for discussion. For all plots 2- $\sigma$ upper limits of $f_{X}$ as derived in Sec. 5 are used.

The dominant effect of energy injection is to damp the CMB anisotropy power spectrum at high $\ell$. For lifetimes longer than recombination epoch $\left(\tau_{X}=10^{14} \mathrm{~s}\right)$, freeze-out ionization fraction is most affected which results in step-function like damping. For lower lifetimes, the recombination history is modified closer to the hydrogen recombination epoch also and results in the $\ell$-dependent damping in power spectra.

\section{Effect of dark matter decay on the $6 \Lambda$ CDM parameters}

The 2-parameter 1- $\sigma$ and 2- $\sigma$ contours are plotted in Fig. 14. We see that there is almost no degeneracy between the $\Lambda$ CDM parameters and energy injection from dark matter decay. This is consistent with the previous results of [30] who consider $\tau_{X} \geq 10^{13} \mathrm{~s}$. As a result there is negligible change in the $6 \Lambda \mathrm{CDM}$ cosmological parameters, the baryon density $\left(\Omega_{\mathrm{b}} h^{2}\right)$, the cold dark matter density $\left(\Omega_{\mathrm{c}} h^{2}\right)$, the angular acoustic scale at recombination $\left(\theta_{\mathrm{MC}}\right)$, the optical depth to reionization $(\tau)$, and the amplitude $\left(A_{\mathrm{s}}\right)$ and spectral index of $\left(n_{\mathrm{S}}\right)$ of primordial fluctuations. This can be seen in Table 3 where we give the mean and 1- $\sigma$ deviation for standard cosmological parameters for different energy injection scenarios and as well as

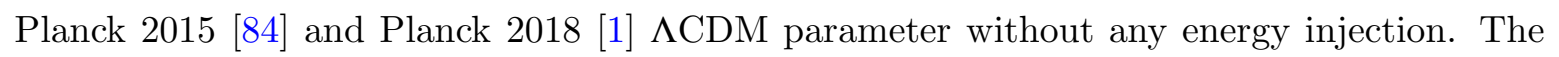
main change from 2015 to 2018 is the improved low $\ell$ polarization data which changes the reionization optical depth. However, since there is no degeneracy between energy injection and $\tau$, we do not expect our constraints, which were derived with 2015 likelihoods, to be affected. 


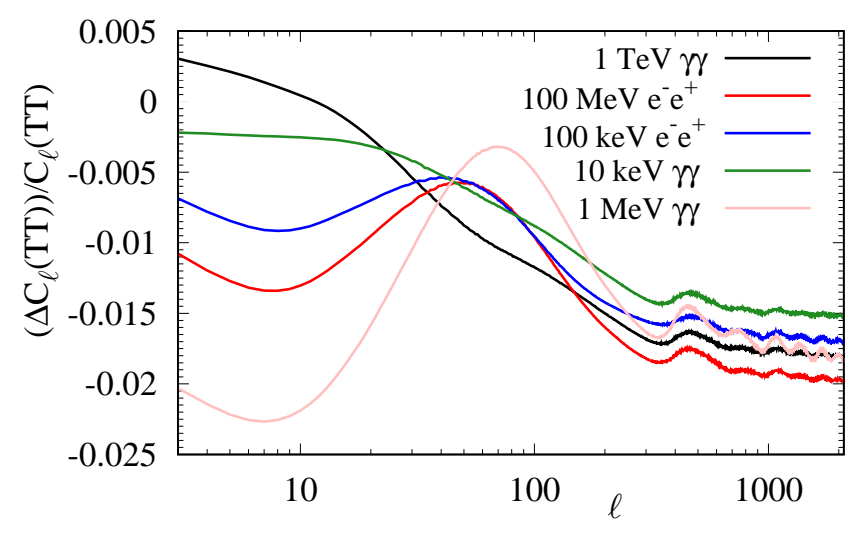

(a) $\tau_{X}=10^{13} \mathrm{~s}$.

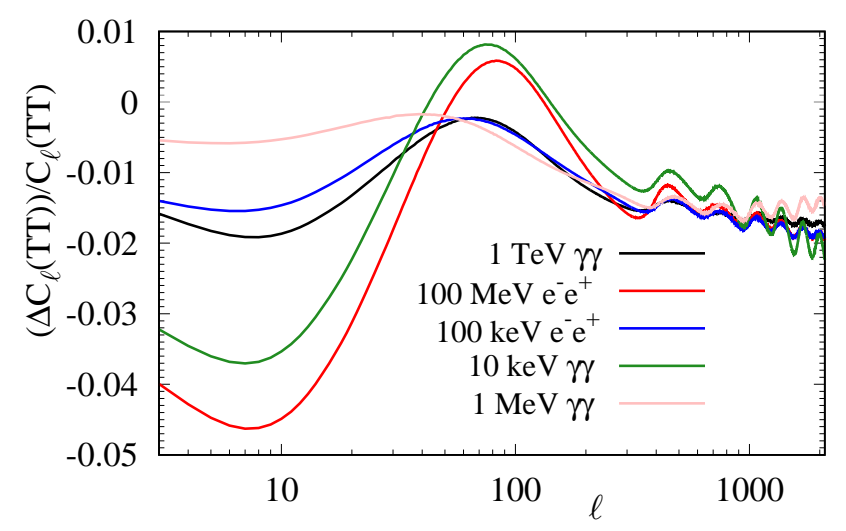

(c) $\tau_{X}=10^{12} \mathrm{~s}$.

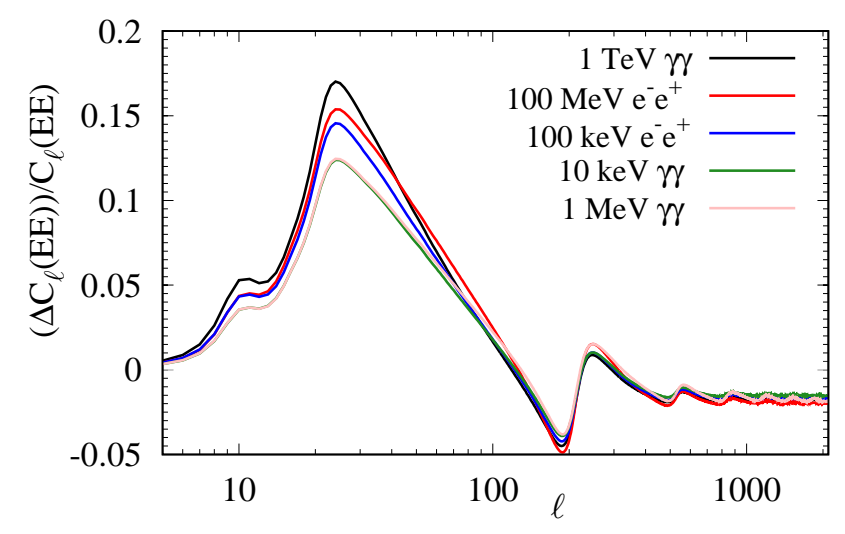

(b) $\tau_{X}=10^{13} \mathrm{~s}$.

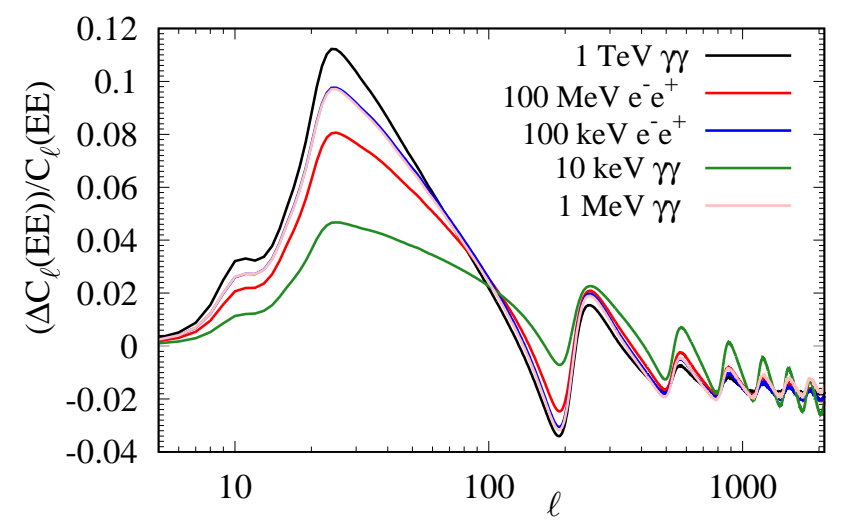

(d) $\tau_{X}=10^{12} \mathrm{~s}$

Figure 12: Fractional change in CMB temperature amd polarizarion power spectrum for different dark matter decay scenarios. 


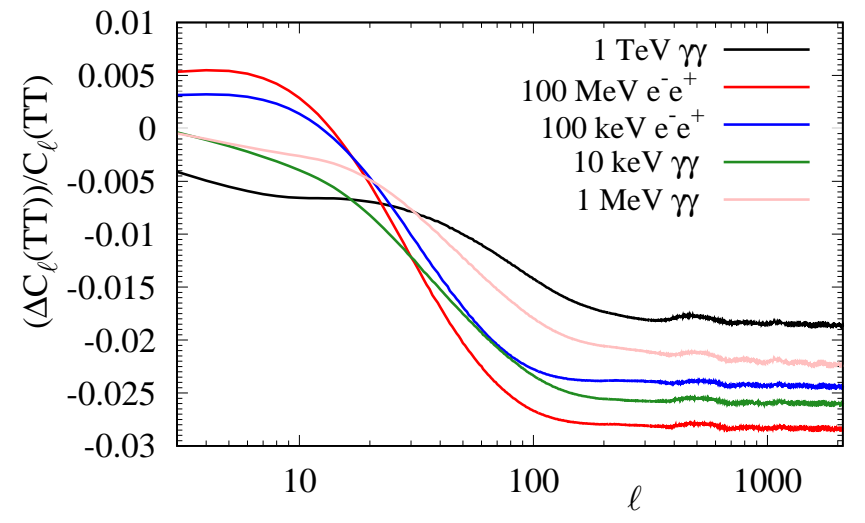

Figure 13: Fractional change in CMB temperature power spectrum for different dark matter decay scenarios for $\tau_{X}=10^{14} \mathrm{~s}$.

Table 3: Mean values with 1- $\sigma$ deviation for standard cosmological parameters with varying energy and lifetime compared to the Planck 2015 [84] and Planck 2018 [1] $\Lambda$ CDM results without energy injection.

\begin{tabular}{l|c|c|c|c|c|c} 
Case $\left(\tau_{X}\right)$ & $\Omega_{b} h^{2}$ & $\Omega_{c} h^{2}$ & $100 \theta_{M C}$ & $\tau$ & $\ln \left(10^{10} A_{S}\right)$ & $n_{S}$ \\
\hline PL2015 & $0.02225 \pm 0.00016$ & $0.1198 \pm 0.0015$ & $1.04077 \pm 0.00032$ & $0.079 \pm 0.017$ & $3.094 \pm 0.034$ & $0.9645 \pm 0.0049$ \\
PL2018 & $0.02236 \pm 0.00015$ & $0.1202 \pm 0.0014$ & $1.04090 \pm 0.00031$ & $0.0544_{-0.0070}^{+0.0081}$ & $3.045 \pm 0.016$ & $0.9649 \pm 0.0044$ \\
$1 \mathrm{TeV}\left(10^{16} s\right)$ & $0.02221 \pm 0.00016$ & $0.1204 \pm 0.0015$ & $1.04066 \pm 0.00032$ & $0.074 \pm 0.017$ & $3.092 \pm 0.032$ & $0.9643 \pm 0.0049$ \\
$1 \mathrm{TeV}\left(10^{14} s\right)$ & $0.02222 \pm 0.00016$ & $0.1203 \pm 0.0015$ & $1.04067 \pm 0.00033$ & $0.075 \pm 0.017$ & $3.093 \pm 0.033$ & $0.9636 \pm 0.0048$ \\
$1 \mathrm{TeV}\left(10^{13} s\right)$ & $0.02221 \pm 0.00017$ & $0.1205 \pm 0.0019$ & $1.04063 \pm 0.00039$ & $0.075 \pm 0.018$ & $3.094 \pm 0.033$ & $0.9638 \pm 0.0049$ \\
$1 \mathrm{TeV}\left(10^{12} s\right)$ & $0.02222 \pm 0.00016$ & $0.1204 \pm 0.0015$ & $1.04067 \pm 0.00034$ & $0.075 \pm 0.017$ & $3.093 \pm 0.033$ & $0.9643 \pm 0.0048$ \\
$100 \mathrm{MeV}\left(10^{16} s\right)$ & $0.02222 \pm 0.00016$ & $0.1202 \pm 0.0015$ & $1.04070 \pm 0.00033$ & $0.072 \pm 0.017$ & $3.097 \pm 0.032$ & $0.9635 \pm 0.0048$ \\
$100 \mathrm{MeV}\left(10^{14} s\right)$ & $0.02219 \pm 0.00021$ & $0.1208 \pm 0.0026$ & $1.04061 \pm 0.00045$ & $0.070 \pm 0.020$ & $3.094 \pm 0.034$ & $0.9631 \pm 0.0051$ \\
$100 \mathrm{MeV}\left(10^{13} s\right)$ & $0.02221 \pm 0.00016$ & $0.1205 \pm 0.0016$ & $1.04064 \pm 0.00034$ & $0.075 \pm 0.017$ & $3.092 \pm 0.033$ & $0.9639 \pm 0.0048$ \\
$100 \mathrm{MeV}\left(10^{12} s\right)$ & $0.02222 \pm 0.00016$ & $0.1204 \pm 0.0015$ & $1.04054 \pm 0.00035$ & $0.077 \pm 0.017$ & $3.095 \pm 0.033$ & $0.9650 \pm 0.0048$ \\
$1 \mathrm{MeV}\left(10^{16} s\right)$ & $0.02222 \pm 0.00016$ & $0.1203 \pm 0.0015$ & $1.04065 \pm 0.00033$ & $0.073 \pm 0.018$ & $3.094 \pm 0.033$ & $0.9635 \pm 0.0048$ \\
$1 \mathrm{MeV}\left(10^{14} s\right)$ & $0.02222 \pm 0.00016$ & $0.1204 \pm 0.0016$ & $1.04064 \pm 0.00035$ & $0.074 \pm 0.018$ & $3.095 \pm 0.032$ & $0.9636 \pm 0.0049$ \\
$1 \mathrm{MeV}\left(10^{13} s\right)$ & $0.02222 \pm 0.00016$ & $0.1205 \pm 0.0016$ & $1.04061 \pm 0.00036$ & $0.077 \pm 0.018$ & $3.095 \pm 0.034$ & $0.9643 \pm 0.0048$ \\
$1 \mathrm{MeV}\left(10^{12} s\right)$ & $0.02221 \pm 0.00016$ & $0.1204 \pm 0.0015$ & $1.04051 \pm 0.00037$ & $0.076 \pm 0.017$ & $3.092 \pm 0.033$ & $0.9634 \pm 0.0047$
\end{tabular}



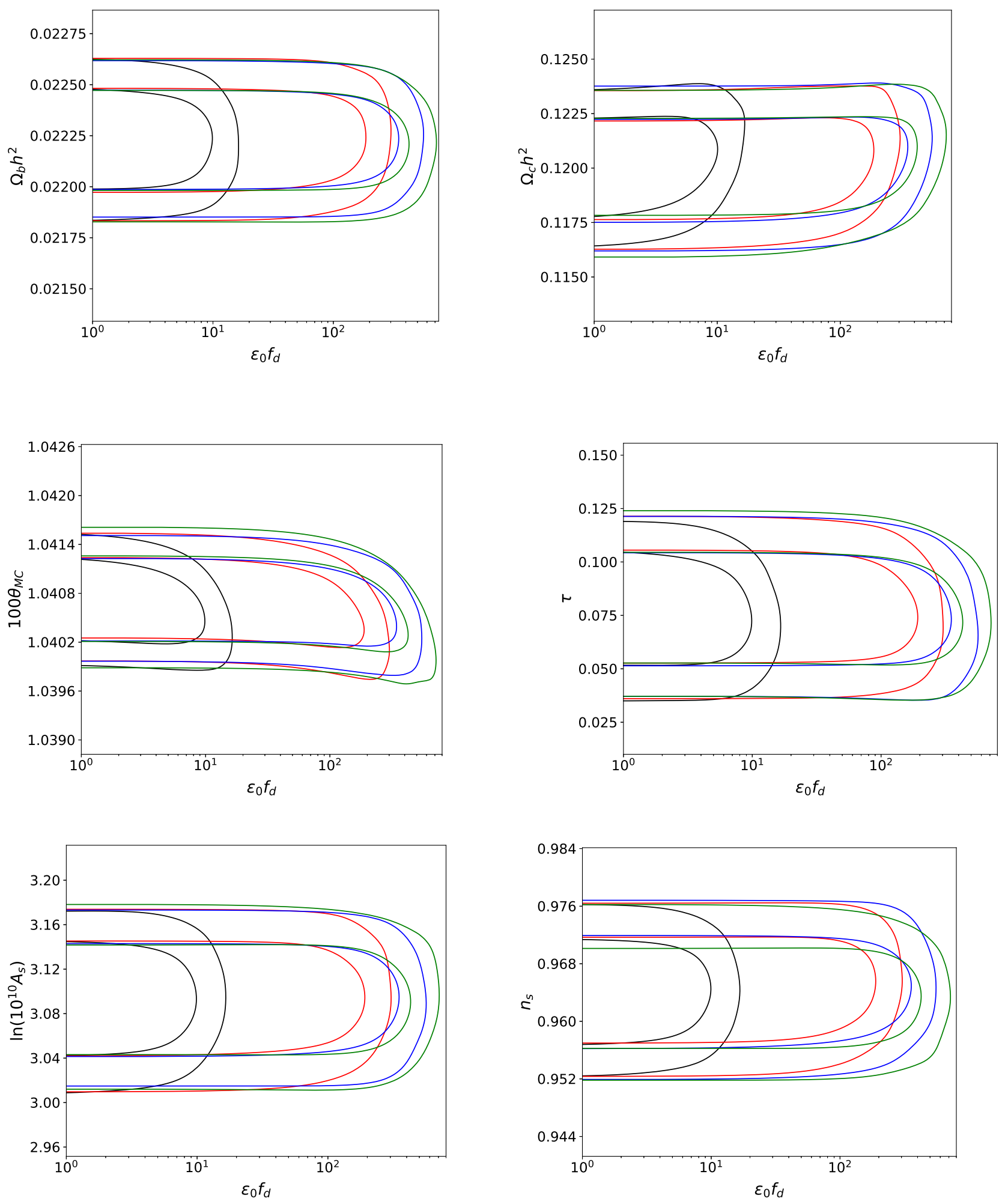

Figure 14: 1- $\sigma, 2-\sigma$ contour for $f_{X}$ and $6 \Lambda \mathrm{CDM}$ parameters for different energy injection scenarios (1 TeV $\gamma \gamma$ (black), $100 \mathrm{MeV} e^{-} e^{+}$(red), $100 \mathrm{keV} e^{-} e^{+}$(blue), $1 \mathrm{MeV} \gamma \gamma$ (green) with $\tau_{X}=10^{12}$ s. $f_{X}$ is related to $\epsilon_{0} f_{d}$ as $f_{X}=\epsilon_{0} f_{d} \times 10^{-10}$. 